\title{
The effects of age on channel capacity for absolute identification of tonal duration
}

\author{
Dana R. Murphy \\ Nipissing University, North Bay, Ontario, Canada \\ BRUCE A. SCHNEIDER \\ University of Toronto at Mississauga, Mississauga, Ontario, Canada \\ AND \\ Helen BaIley \\ Nipissing University, North Bay, Ontario, Canada
}

\begin{abstract}
We compared the ability of younger and older adults to identify which 2-kHz tones of eight varying durations was presented on a trial with their ability to discriminate between adjacent pairs of duration-varying tones drawn from the same set. We used signal detection analyses to construct scales of perceived duration for both tasks. Scales derived from pairwise comparisons of adjacent durations were related linearly to the logarithm of stimulus duration; these were essentially identical in younger and older adults. However, scales derived from the eight-alternative absolute identification experiments, which were also linearly related to the logarithm of duration, indicated that older adults outperformed younger adults on this task. These results suggest that the ability to process large numbers of stimuli that differ only in duration is at least as good, if not better, in older than in younger adults, relative to the ability of each group to discriminate between two stimuli differing only in duration.
\end{abstract}

To extract and process attended stimuli when the listening situation is poor (e.g., when there are competing sound sources or there is excessive reverberation), listeners often need to engage higher order, more central processing mechanisms. By focusing their attention on particular frequency regions, by inhibiting irrelevant signals, and by using context to disambiguate signals, listeners are often able to recover information that is degraded or lost at the sensory level. Because of age-related declines in hearing, older adults are more likely than younger adults are to have to engage these higher order processes in order to function in everyday listening situations (see Schneider, PichoraFuller, \& Daneman, 2009, for a review). Consequently, they may become more skilled at operating under such conditions than younger adults, provided that the relevant higher order central processes are preserved in old age. Indeed, there is evidence that older adults benefit more from context than do younger adults in difficult listening situations (Pichora-Fuller, Schneider, \& Daneman, 1995; Sheldon, Pichora-Fuller, \& Schneider, 2008), suggesting that, not only is the ability to use context to disambiguate signals preserved in old age, but older adults become more skilled in its employment.

Given that there are large and obvious declines with age in peripheral auditory processes (Murphy, Schneider, Speranza, \& Moraglia, 2006; Schneider \& Pichora-Fuller,
2000), it also may be that some higher order or more central auditory and cognitive processes decline with age. If they do, the auditory processing abilities of older adults would be doubly compromised - first, by cochlear degeneration, and second, by age-related changes in retrocochlear auditory processes. Although there is considerable evidence that age-related cochlear degeneration greatly reduces the spectral and temporal resolution of the auditory system (see Schneider, 1997; Schneider \& Pichora-Fuller, 2000, 2001, for recent reviews), thereby compromising the signal at the level of the cochlea, the evidence concerning age-related changes in retrocochlear processes in healthy aging is sparse. For example, Ison, Virag, Allen, and Hammond (2002) and Murphy et al. found no evidence of age-related changes in auditory attention bands - that is, changes in the ability of older adults to focus their attention on a particular frequency range. In addition, Murphy et al. failed to find any evidence for age-related declines in top-down gain control - that is, in the ability of the auditory system to adjust its gain to accommodate different listening situations. A number of studies have shown that there is a nonlinear amplifier within the auditory system that amplifies very low-intensity stimuli and attenuates high-intensity stimuli, thereby extending the range over which the auditory system can respond (Brownell, 1997; Gordon \& Schneider, 2007; Parker, Murphy, \& Schneider,

D. R. Murphy, danam@nipissingu.ca 
2002; Robles \& Ruggero, 2001; Yates, 1995). Loss of control over this nonlinear amplifier would greatly limit the ability of the auditory system to function over a wide range of amplitudes. However, Murphy et al. found no evidence that top-down control over this nonlinear amplifier diminished with age. Hence, older adults appear to be as good as younger adults are at adjusting auditory gain to optimize signal processing.

\section{Age-Related Changes in Channel Capacity}

The evidence is mixed regarding age-related declines in a third retrocochlear process - the ability to identify simple stimuli. Miller (1956) showed that the ability of listeners to identify stimuli varying along a single dimension is limited by that dimension's channel capacity. Murphy et al. (2006) measured the channel capacities of younger and older adults by having them identify pure tones that differed only in intensity. To avoid problems associated with age-related sensory declines, they adjusted the intensity differences between adjacent tones to be nearly perfectly discriminable to both younger and older adults. Otherwise, the cochlear degeneration caused by presbycusis may have diminished the ability of older adults to identify the tones at a peripheral level, thereby compromising the measurement of channel capacity per se. With tones spaced $6 \mathrm{~dB}$ apart, no age-related differences were found in the ability to identify between two and eight pure tones. Hence, with respect to stimulus intensity, channel capacity appears to be well preserved with age.

McCormack, Brown, Maylor, Richardson, and Darby (2002), on the other hand, reported that older adults were less accurate than were younger adults in identifying pure tones that varied only in duration. However, the tonal durations used by these investigators were such that some of the pairs of adjacent stimuli were likely to be below the discrimination thresholds of older adults (Bergeson, Schneider, \& Hamstra, 2001). Hence, the poorer performance of older adults in identifying tones differing only in duration found by McCormack et al. (2002) could have reflected the inability of older adults to distinguish between pairs of adjacent stimuli, rather than an agerelated diminution in the channel capacity for stimulus duration.

The results of the McCormack et al. (2002) study are consistent with those from a large number of studies that indicate that general timing abilities, including the verbal estimation of a specified length of time and the ability to reproduce a previously presented interval of a specific duration, decline with age (Block, Zakay, \& Hancock, 1998; Craik \& Hay, 1999; Perbal, Droit-Volet, Isingrini, \& Pouthas, 2002; Wearden, Wearden, \& Rabbitt, 1997). For instance, in their meta-analysis of a large number of studies comparing younger and older adults on tasks requiring time reproduction and time production, Block et al. found substantial age-related changes in these abilities. When asked to produce an interval of a given duration, older adults typically produce a longer interval than younger adults do. On the other hand, when they are asked to reproduce an interval that they have just experienced, older adults reproduce a shorter interval than younger adults do.
Age-related changes in the production or reproduction of intervals, however, could occur without changes in the channel capacity for the time sense. Older adults may simply perceive intervals of time to be shorter (or, in some cases, longer; Craik \& Hay, 1999) than do their younger counterparts, but they could be equally adept at discriminating among a set of such intervals. Moreover, in an identification experiment, response biases could influence the performance of older participants in a way that is unrelated to channel capacity. A second reason for conducting the present experiment, in addition to controlling for age-related difference in pairwise discriminablity, was to assess the extent to which age differences in response bias could produce the age-related differences in performance that are sometimes found in identification experiments (e.g., McCormack et al., 2002).

\section{Models of Duration Discrimination}

The absolute identification of stimulus duration can be modeled in different ways. In a signal detection model of duration discrimination, repeated presentations of a stimulus of fixed duration elicit a distribution of duration responses along a decision axis. Figure 1 illustrates hypothetical distributions corresponding to two stimuli (S1 and S2). Although the distributions in signal detection theory (SDT) usually are assumed to be equal-variance normal distributions, here the distributions are assumed to have equal variance but to be Laplacian in shape for two reasons. First, Schneider (2007) has shown that, even when the response matrix in an absolute identification experiment is generated by a model having equal-variance normal distributions, an equal-variance Laplace signal detection model provides a better fit to the data under certain circumstances. Specifically, if the standard deviation $(S D)$ of the underlying normal distributions were to vary randomly over a session (or vary between participants, when the response matrices from different participants are averaged), the resulting data matrices would be better fit by equal-variance Laplacian than by equal-variance normal

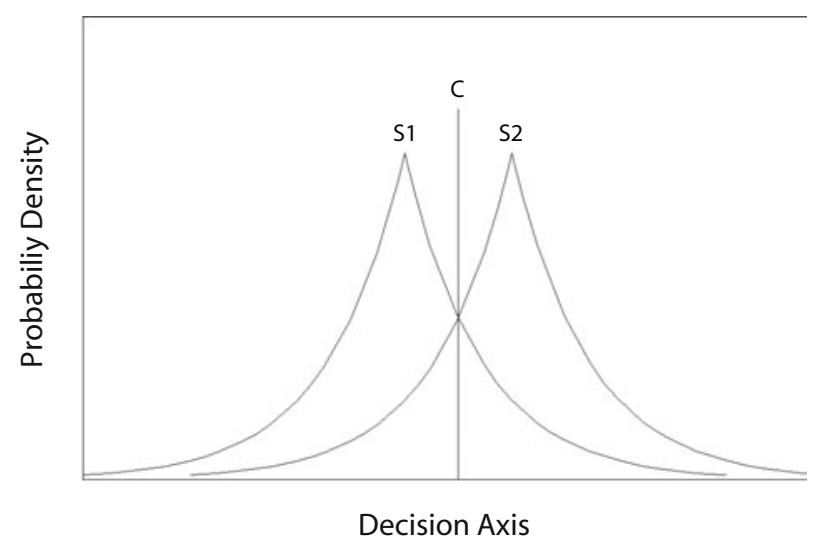

Figure 1. Hypothetical distributions of responses elicited by presentations of either Stimulus 1 (S1) or Stimulus 2 (S2) along a unidimensional decision axis. Observations to the left of the criterion are identified as Stimulus 1; those to the right are identified as Stimulus 2. 
distributions, even though the data were generated from normal distributions. Second, Parker et al. (2002) have shown that data from absolute judgments of tones varying only in intensity are better fit by a Laplace model than by a normal signal detection model.

Figure 1 also shows a criterion that separates the decision axis into two response regions. If the presentation of a stimulus gives rise to a response along the decision axis that is to the left of the criterion, it is assumed that the observer will respond "S1" and otherwise respond "S2." The measure of discriminability, called $d^{\prime}$, is the separation of the means of the two distributions divided by their $S D$. Because these are Laplace distributions, we will refer to this measure of discriminability as $d_{\mathrm{L}}^{\prime}$. The value of $d_{\mathrm{L}}^{\prime}$ is obtained, as usual, from hits ( $\mathrm{S} 2$ is correctly identified as $\mathrm{S} 2$ ) and false alarms ( $\mathrm{S} 1$ is incorrectly identified as $\mathrm{S} 2$ ). (See Macmillan \& Creelman, 2005, for a more detailed account of SDT.) Because Brown, McCormack, Smith, and Stewart (2005) have argued that the type of effects (e.g., stimulus range effects and bias effects) found in absolute identification experiments of stimulus duration are equivalent to those found when other stimulus dimensions (such as tonal intensity) are employed, it seems reasonable to use SDT theory, which has been applied successfully to analyze absolute judgments of other stimulus dimensions (e.g., loudness; Durlach \& Braida, 1969; Parker et al., 2002), to analyze absolute judgments of tonal duration.

A second and quite popular model of duration discrimination derives from scalar expectancy theory (SET). In SET (see Allan, 1998), it is assumed that, at the beginning of the interval, the observer starts an internal clock that accumulates counts at a more or less fixed rate. The clock is then switched off at the end of the interval, and the observer's judgment of time is based on a comparison of the clock count and a memory representation of the interval's duration (also based on clock counts). In some models of this process, it is assumed that the clock count is accurate (no variability in clock rate), but that there is some variability in the memory representation $(m)$ of that interval (see, e.g., Wearden et al., 1997). Hence, the output of this comparison process, relative to the true duration $(t)$ of the interval, is assumed to be a random variable, $t-m$, which varies randomly from trial to trial due to random variations in the remembered value, $m$, of the duration of the interval. The random variable $m$ is assumed to have normal distribution with $M=t-D$ and $S D=a *(t-D)$. Note that, if $D=$ 0 (the usual assumption that there is no bias in the remembered location), the mean of the memory variable is equal to the true duration and the $S D$ of the memory variable is proportional to the mean. In this case, $t-m$ is a normally distributed variable, with $M=0$ and $S D=a * t$.

One version of SET theory (Wearden, 2004) assumes that the decision as to whether a particular count is an example of the interval is based on the criterion

$$
\frac{\operatorname{Abs}(t-m)}{t} \text {. }
$$

If the value of this decision variable is less than some criterion, the response would be "Yes"; this is an example of the interval $t$. If the value of the decision variable is greater than this criterion, the response would be "No." This decision rule can be extended to the absolute judgment case in the following manner.

Assume that eight intervals could be presented on a trial. When an interval is presented, the observer starts a clock count that terminates at the end of the presented interval and then compares this count with the memory of each of the eight intervals to obtain a measure of how close the clock count comes to the remembered value of the clock count for each remembered interval. That is, for an interval of length $t$, the observer computes

$$
\frac{\operatorname{Abs}\left(t-m_{1}\right)}{t}, \frac{\operatorname{Abs}\left(t-m_{2}\right)}{t}, \frac{\operatorname{Abs}\left(t-m_{3}\right)}{t}, \ldots, \frac{\operatorname{Abs}\left(t-m_{8}\right)}{t}
$$

The observer then identifies the interval as the one whose decision variable has the lowest value. Because the $m \mathrm{~s}$ are random variables, sometimes the interval $t$ will be misidentified. See Appendix A for further details of this model.

Another version of SET theory, which deals directly with absolute identification of durations and is elaborated in McCormack et al. (2002), assumes that the psychological similarity between the duration stimulus $S_{i}$ being presented and the remembered value of another stimulus $S_{j}$ is

$$
\eta_{i, j}=e^{-c\left|\log _{e}\left(\frac{S_{i}}{S_{j}}\right)-D\right|},
$$

where $D$ represents the degree to which the remembered duration of stimulus $S_{j}$ is distorted in memory. Other investigators, in addition to McCormack et al. (2002), have argued that, within a SET model, a nonzero memory distortion parameter $D$ is necessary to fully account for differences across age groups (e.g., Wearden et al., 1997) or for the effects of pharmacological manipulations on performance in animals (Meck, 1996). Note that this model assumes that the representation of stimulus duration in memory is logarithmically related to stimulus duration and that, unlike the previous SET model, the remembered duration can be biased (for details, see Appendix A). Hence, in addition to our evaluation of the ability of SDT to account for our obtained results, we also tested this version of SET to determine whether the addition of a memory parameter would provide a better account of the data than would the standard SET model mentioned earlier. Thus, in this research, in addition to our evaluation of SDT models, we also evaluated the fit of two SET models to our obtained data in order to determine which of these two SET-based models provided a better fit to the absolute identification of tonal durations and to compare these models with the SDT model.

\section{The Present Research}

To determine whether the reduced channel capacity for stimulus duration that McCormack et al. (2002) observed in older adults was due to age-related reductions in the ability to discriminate duration differences or to age-related differences in response bias, we decided to replicate their study using two different sets of logarithmically spaced stimuli, with the spacing between adjacent 
stimuli being larger in one set than in the other, but not so large that adjacent pairs were perfectly discriminable. To permit comparison of pairwise discriminability with performance in the absolute identification experiment, we also measured the discriminability of all adjacent pairs of stimuli from the identification experiment.

To do so, we tested younger and older adults on their ability to identify pure tones differing only in duration in an absolute identification paradigm for two different sets of eight durations. To assess the degree to which the discriminability of adjacent pairs of stimuli affected performance in the eight-alternative identification experiment, we also measured the discriminability of adjacent pairs of stimuli and related this to performance in the absolute identification experiment.

Different groups of younger and older adults completed the task in Experiment 1 and Experiment 2 using different duration sets in each experiment. However, given the similarity between the task procedures in both, we have chosen to provide the experimental details in single Method and Results sections to minimize repetition of identical details and to facilitate the comparison across experiments.

\section{METHOD}

\section{Participants}

In Experiment 1, 17 younger adults (11 women) and 17 older adults ( 12 women) participated. The younger adults ranged in age from 18 to 25 years $(M=20.35, S D=1.73)$, scored an average of $12.18(S D=4.16)$ on the Mill Hill Vocabulary Test, and had completed an average of 14.47 years of education $(S D=3.17)$. The older adults ranged in age from 60 to 78 years $(M=69.82, S D=4.86)$, scored an average of $15.65(S D=2.34)$ on the Mill Hill Vocabulary Test, and had completed an average of 14.47 years of education $(S D=3.11)$. This difference on the vocabulary score, in favor of the older adults, was significant $[t(32)=2.99, p<.005]$.

In Experiment 2, 28 younger adults (16 women) and 28 older adults (20 women) participated. The younger adults ranged in age from 17 to 28 years $(M=20.79, S D=2.64)$, scored an average of 13.96 $(S D=1.99)$ on the Mill Hill Vocabulary Test, and had completed an average of 15.71 years of education $(S D=2.14)$. The older adults ranged in age from 60 to 80 years $(M=68.79, S D=5.36)$, scored an average of $14.96(S D=2.36)$ on the Mill Hill Vocabulary Test, and had completed an average of 12.84 years of education $(S D=3.62)$. Although there was no difference between the two groups in terms of their vocabulary scores $[t(54)=1.72, p>.09]$, the younger adults had completed significantly more education than had the older adults $[t(51)=3.56, p<.001]$. All participants were from the greater North Bay area. The younger adults were students of Nipissing University, and the older adults were members of the general North Bay community. All were paid $\$ 10 / \mathrm{h}$ (Canadian) for their participation.

The hearing for all participants was screened using pure-tone audiometry. Figure 2 presents the average thresholds for the younger and older adults in Experiments 1 and 2 for the left ear as a function of frequency. A 2 (age: young, old) $\times 9$ (frequency: 250, 500, $1000,1500,2000,3000,4000,6000$, and $8000 \mathrm{~Hz}$ ) ANOVA showed a significant age main effect $\left[F(1,88)=136.75, M S_{\mathrm{e}}=587.93\right.$, $p<.001]$, a significant frequency main effect $[F(8,704)=79.79$, $\left.M S_{\mathrm{e}}=80.56, p<.001\right]$, and a significant age $\times$ frequency interaction $\left[F(8,704)=49.67, M S_{\mathrm{e}}=80.56, p<.001\right]$. The ANOVA results indicate that the older adults in the present study had significantly higher deficits in hearing sensitivity at all frequencies, with the degree of deficit increasing with frequency. Thresholds for all the younger adults were well within the normal range. However, the audiometric thresholds of the older adults indicated that they were in the early stages of presbycusis.

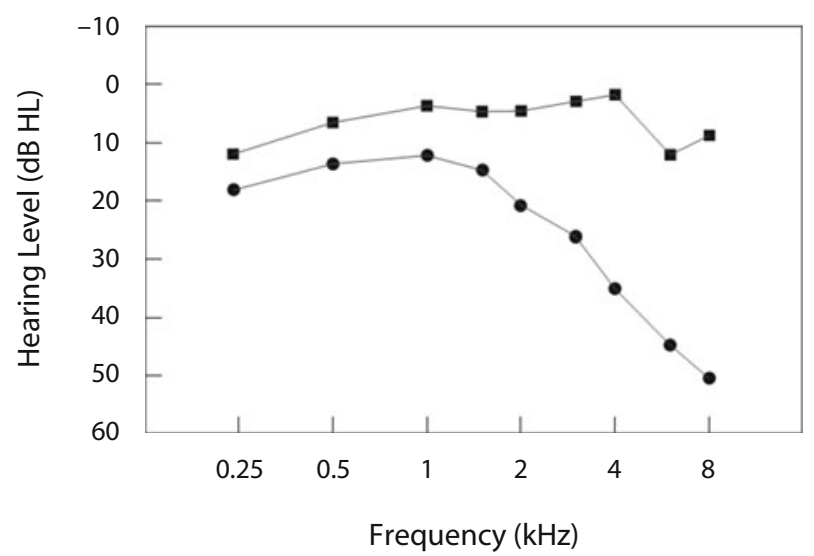

Figure 2. Audiometric thresholds as a function of frequency for the young (squares) and old (circles) participants averaged across Experiments 1 and 2 for the left ear.

We also administered a babble threshold test, which calculates the threshold of hearing when the stimulus is a 12-talker babble stimulus (a recording of 12 people talking simultaneously; Bilger, Nuetzel, Rabinowitz, \& Rzeczkowski, 1984). Participants were asked to identify the interval in a two-interval, forced choice paradigm containing the babble. No sound was presented in the other interval. This test used a 3-down 1-up staircase procedure to identify the $79 \%$ threshold for babble speech on the psychometric function (Levitt, 1971). In this procedure, the intensity at which the babble was presented was reduced by the maximum step size following three correct responses, and upon the first incorrect response, the intensity was increased and the step size decreased. The participant's threshold for babble speech was calculated as the average intensity value of the last eight reversals. Participants with babble thresholds in excess of $35 \mathrm{~dB}$ sound pressure level (SPL) were not included in these experiments. Volunteers who did not meet these criteria were not invited to participate in this study. On average, approximately $28 \%$ of the older participants whose hearing are tested in this fashion are excluded from studies in the Northern Centre for this reason. Younger adults $(M=20.49 \mathrm{~dB}, S D=3.57 \mathrm{~dB})$ had significantly lower babble thresholds on average than did older adults $[M=29.43 \mathrm{~dB}, S D=$ $5.42 \mathrm{~dB} ; t(88)=9.24, p<.001]$.

\section{Apparatus and Stimuli}

The stimuli were constructed in the same way as those used in the Bergeson et al. (2001) study. As a review, a 16-bit, 2-kHz pure tone was generated digitally with a sampling rate of $20 \mathrm{kHz}$. We gated the $2-\mathrm{kHz}$ tone on and off by multiplying it by an envelope constructed by summing a series of Gaussian envelopes $(S D=0.5 \mathrm{msec})$, spaced $0.5 \mathrm{msec}$ apart. The duration of the stimulus was defined as the time between the centers of the first and last Gaussian envelopes, which in each of the stimuli employed here were at the one half power points of the envelope. Therefore, stimulus duration is the difference between the one half power points of the envelope. For stimuli $\geq 400 \mathrm{msec}$, the SPL of the stimulus during its steady-state portion was $66.5 \mathrm{~dB}$. For stimuli $<400 \mathrm{msec}$, the total energy in the stimulus was set equal to the total energy in the 66.5-dB SPL, 400msec stimulus. Thus, stimuli $<400 \mathrm{msec}$ were equated for energy, whereas stimuli $\geq 400 \mathrm{msec}$ were equated for SPL. Short-duration stimuli were equated for total energy because of the intensity/time trade-off and to minimize spectral differences between tones of different durations.

Later, the same stimuli were resampled to a rate of $24.414 \mathrm{kHz}$ using the GoldWave digital audio editing program. When presented to the participant, the signals were generated and converted from digital to analog using the RP2.1 unit of the Tucker-Davis Technologies (TDT) System III hardware. The signal was then attenu- 
ated using a TDT programmable PA5 unit, routed through a TDT headphone buffer, and presented to the participant through the left headphone of TDH-39 headphones.

The eight durations in Experiment 1 (220, 280, 355, 430, 520, 620,730 , and $850 \mathrm{msec}$ ) were spaced more closely than were those in Experiment $2(220,300,410,560,750,1,000,1,310$, and $1,700 \mathrm{msec}$ ). All participants were tested individually in a singlewalled sound-attenuating chamber, and all stimuli were presented through TDH-39 headphones.

\section{Procedure}

Before being admitted into the experiment, participants completed basic pretesting measures that included a hearing and language history form, the Mill Hill Vocabulary Test, audiometric pure tone tests, and a babble threshold test as part of a screening battery given to all participants. On the day of testing, the seven pairwise discriminations between adjacent stimuli were tested first, followed by the absolute identification of all eight tonal durations. Participants were tested in this order to ensure that they could discriminate to some degree between adjacent tonal durations and to give them some exposure to the task and stimuli, using the easier of the two paradigms (pairwise comparisons) before moving on to the more difficult task (absolute identification of eight tonal durations). Breaks were scheduled periodically to prevent fatigue.

Pairwise discrimination thresholds were obtained for pairs of adjacent stimuli by using the absolute identification paradigm with only two stimuli. On a trial, one member of an adjacent pair (e.g., either the 220 - or the 280 -msec stimulus) was presented. Participants were asked to press Button 1 when the shorter duration $(220 \mathrm{msec})$ stimulus was presented and to press Button 2 when the longer duration $(280 \mathrm{msec})$ stimulus was presented. After each response, feedback was provided by illuminating an LED above the button corresponding to the tone duration that was presented. Participants could take as long as they needed to make a response. The next tone was presented $1,500 \mathrm{msec}$ after the participants had responded.

Stimulus pairs were presented in 120 -trial blocks. In order to minimize any practice effects that may have occurred, the order of completion of the seven pairs was controlled through randomization; to do this, we compiled 20 different randomizations of the seven pairs so that no more than 2 participants from each age group completed the pairs in the same order. In each block of 120 trials, participants first completed 20 practice trials (10 short and 10 long stimulus pre- sentations randomly distributed across the 20 practice trials), which were followed by two blocks of 50 test trials. The 50 presentations of each of the two tones were placed randomly throughout the total trials. A set of flashing lights indicated the end of the 20 practice trials and the end of each 50-trial block. Before pressing a button to go on to the next 50-trial block, participants were given the option of taking a break. After each 120-trial block, there was a forced break of approximately $1 \mathrm{~min}$ to set up for the next 120 -trial block. At this point, participants could also take a longer break before proceeding to the next block of trials. Most chose not to do so. All stimuli were presented at $50 \mathrm{~dB}$ above each individual's babble threshold. Participants normally took just over $1 \mathrm{~h}$ to complete this portion of the experiment, with the older adults requiring 3-4 min more, on average, than the younger adults took.

During their third and final testing session, participants were asked to identify which one of eight tonal durations was presented on a trial by pressing the corresponding button on the box (Buttons $1-8$ ). Feedback was again provided by illuminating an LED placed above the button corresponding to the presented tone. This part consisted of 80 practice trials (10 presentations of each stimulus) plus eight blocks of 50 test trials for a total of 400 trials (50 presentations of each stimulus). The 50 presentations of each stimulus were distributed randomly across all 400 possible trials. An unlimited response interval was employed. The next tone was presented 1,500 msec after the participants had given their response. Participants were instructed that they could take a break after each 50-trial block. These breaks were clearly identified to the participants by flashing all of the feedback lights simultaneously. After the break, participants pressed a button to start the next 50-trial block. Most participants chose to move on to the next 50-trial block, either after only a very short break or immediately without a break. All stimuli were presented at $50 \mathrm{~dB}$ above each participant's babble threshold. Participants normally completed this absolute identification portion of the experiment in $36 \mathrm{~min}$, although some participants took more breaks, which extended the time to complete the experiment.

\section{RESULTS}

\section{Pairwise Discriminability}

Table 1 displays the $d_{\mathrm{L}}^{\prime}$ values obtained from group data for adjacent pairs of stimuli in Experiments 1 and 2. If

Table 1

Pairwise Discriminability $\left(d_{\mathrm{L}}^{\prime}\right)$ for Adjacent Stimuli, and Concatenated $d_{\mathrm{L}}^{\prime}$ Values of the Stimuli Along the Decision Axis Obtained From the Group Data for the Young and Old Adults in Experiments 1 and 2

\begin{tabular}{|c|c|c|c|c|c|c|}
\hline \multirow{2}{*}{$\begin{array}{c}\text { Stimulus } \\
\text { Pair }\end{array}$} & \multicolumn{3}{|c|}{ Young } & \multicolumn{3}{|c|}{ Old } \\
\hline & $d_{\mathrm{L}}^{\prime}$ & Concatenated $d_{\mathrm{L}}^{\prime}$ & Duration & $d_{\mathrm{L}}^{\prime}$ & Concatenated $d_{\mathrm{L}}^{\prime}$ & Duration \\
\hline \multicolumn{7}{|c|}{ Experiment 1} \\
\hline & & 0.00 & 220 & & 0.00 & 220 \\
\hline $220-280$ & 1.09 & 1.09 & 280 & 1.21 & 1.21 & 280 \\
\hline $280-355$ & 1.24 & 2.33 & 355 & 1.40 & 2.61 & 355 \\
\hline $355-430$ & 0.98 & 3.31 & 430 & 1.02 & 3.63 & 430 \\
\hline $430-520$ & 1.00 & 4.31 & 520 & 0.94 & 4.57 & 520 \\
\hline $520-620$ & 0.85 & 5.16 & 620 & 0.74 & 5.32 & 620 \\
\hline $620-730$ & 0.62 & 5.78 & 730 & 0.83 & 6.15 & 730 \\
\hline $730-850$ & 0.67 & 6.45 & 850 & 0.67 & 6.82 & 850 \\
\hline \multicolumn{7}{|c|}{ Experiment 2} \\
\hline & & 0.00 & 220 & & 0.00 & 220 \\
\hline $220-300$ & 1.97 & 1.97 & 300 & 1.71 & 1.71 & 300 \\
\hline $300-410$ & 2.56 & 4.54 & 410 & 2.11 & 3.83 & 410 \\
\hline $410-560$ & 2.24 & 6.78 & 560 & 1.85 & 5.68 & 560 \\
\hline $560-750$ & 1.99 & 8.66 & 750 & 1.92 & 7.59 & 750 \\
\hline $750-1,000$ & 2.26 & 11.03 & 1,000 & 1.68 & 9.27 & 1,000 \\
\hline $1,000-1,310$ & 2.26 & 13.29 & 1,310 & 1.95 & 11.22 & 1,310 \\
\hline $1,310-1,700$ & 2.36 & 15.65 & 1,700 & 1.92 & 13.14 & 1,700 \\
\hline
\end{tabular}


stimulus duration varies along only a single psychological dimension, these $d_{\mathrm{L}}^{\prime}$ values can be concatenated to form a subjective scale of stimulus duration by assigning 0 to the shortest duration, the value of $d_{\mathrm{L}, 1,2}^{\prime}$ to the second shortest duration, the value of $d_{\mathrm{L}, 1,2}^{\prime}+d_{\mathrm{L}, 2,3}^{\prime}$ to the third duration, . . , and the value of $d_{\mathrm{L}, 1,2}^{\prime}+d_{\mathrm{L}, 2,3}^{\prime} \ldots+d_{\mathrm{L}, 7,8}^{\prime}$ to the longest duration, where $d_{\mathrm{L}, k, j}^{\prime}$ stands for the $d_{\mathrm{L}}^{\prime}$ value separating durations $k$ and $j$. The concatenated $d_{\mathrm{L}}^{\prime}$ values for Experiments 1 and 2 are also listed in Table 1. Figure 3 plots these concatenated values as a function of log stimulus duration for the younger and older adults in Experiments 1 and 2, along with their best-fitting straight lines (method of least squares). Figure 3 shows that the spacing along the decision axis determined from pairwise discriminability is a logarithmic function of stimulus duration (average squared correlation coefficient, $r^{2}=.998$ for both the young and old participants in Experiment 1

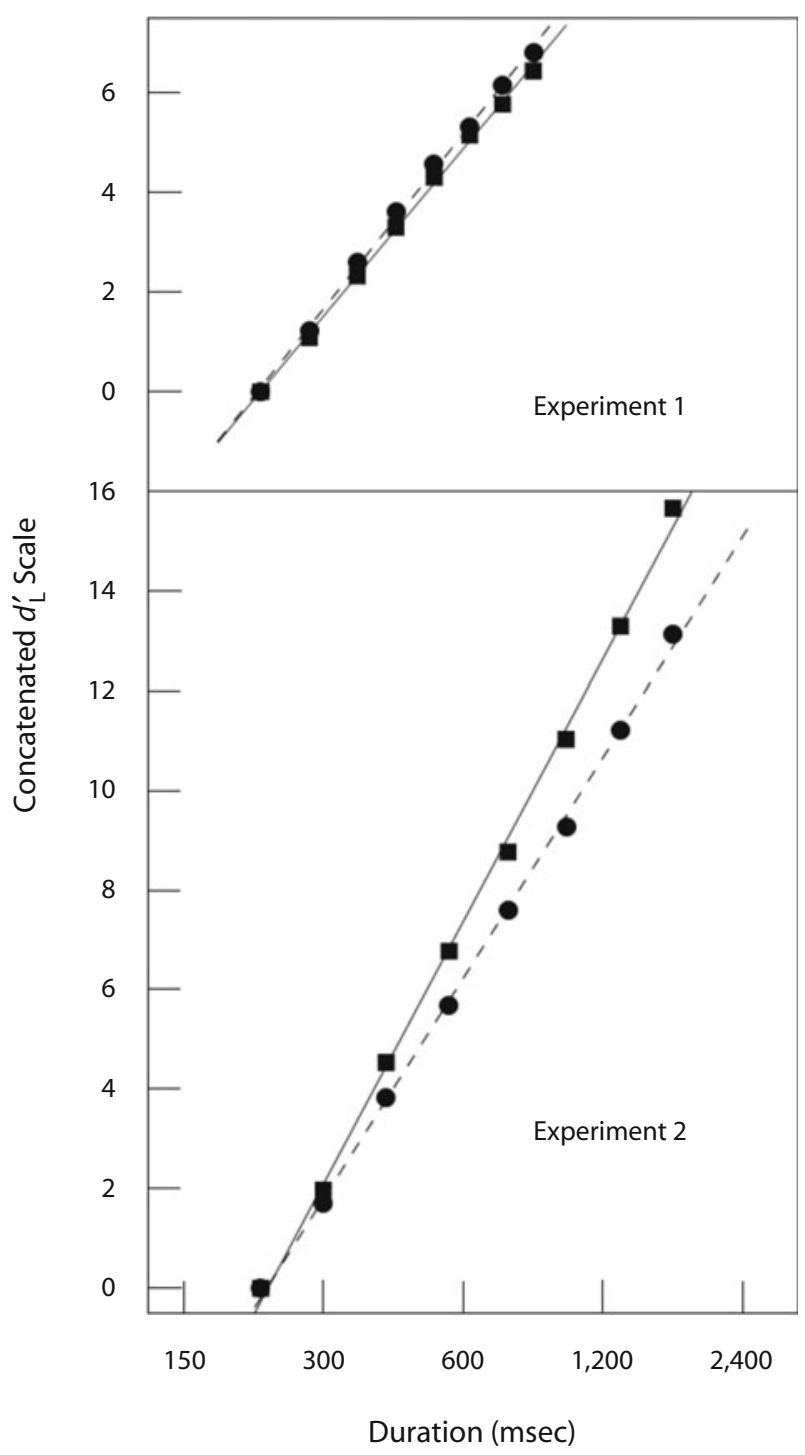

Figure 3. Concatenated $d_{\mathrm{L}}^{\prime}$ (see text) as a function of stimulus duration for the young (squares) and old (circles) participants in Experiments 1 and 2.

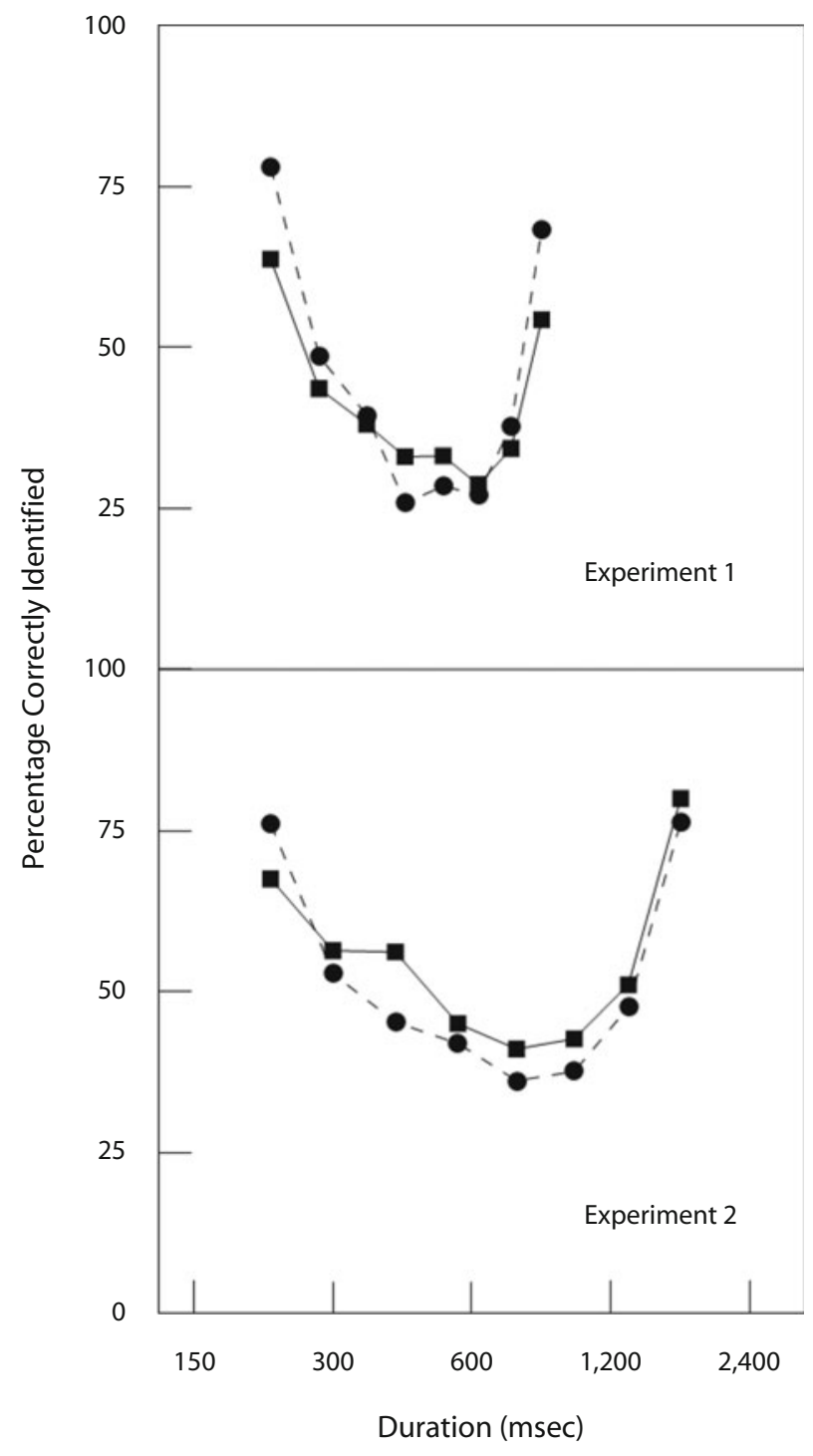

Figure 4. Percentage of stimuli correctly identified as a function of their duration for young (squares) and old (circles) participants in Experiments 1 and 2.

and for the young participants in Experiment 2, and .999 for the old participants in Experiment 2). Figure 3 also shows that the concatenated $d_{\mathrm{L}}^{\prime}$ values are virtually identical for the younger and older adults in Experiment 1, but not in Experiment 2, where the slope of the line relating concatenated values to log duration is steeper (i.e., better discrimination) for younger than for older adults.

To test whether the slopes of these straight lines differed significantly between the young and old groups in these two experiments, we also determined the concatenated $d_{\mathrm{L}}^{\prime}$ scales for each participant in the two experiments and plotted them against log duration. Straight lines provided a good fit to each of these 90 plots $\left(r^{2}=.98\right.$ for both the young and old participants in Experiment 1 and for the old participants in Experiment 2, and .99 for the young participants in Experiment 2). A 2 (age: young, old) $\times$ 2 (experiment: Experiment 1, Experiment 2) ANOVA 
on the logarithms of the slopes ${ }^{1}$ showed a significant effect of experiment $\left[F(1,86)=16.90, p<.0001, \eta_{\mathrm{p}}^{2}=\right.$ .16], but no significant effect of age $[F(1,86)<1]$ and no significant age $\times$ experiment interaction $[F(1,86)=$ $\left.1.72, p>.15, \eta_{\mathrm{p}}^{2}=.02\right]$. Hence, there is no statistical evidence that the young and the old differ in terms of their ability to discriminate pairs of adjacent durations, even though the mean data suggest that, in Experiment 2, the younger adults had better temporal acuity than did the older adults.

\section{Absolute Identification of Duration \\ Identification accuracy. Figure 4 plots percentage of correct identifications as a function of stimulus du- ration for young and old observers when the durations were spaced close together (Experiment 1, top panel) and when they were spaced further apart (Experiment 2, bot- tom panel). In both experiments, older adults performed better than younger adults did for the shortest duration, but worse than younger adults performed at intermedi-}

ate durations. At the longest duration, older adults performed better than younger adults in Experiment 1, but slightly worse than younger adults in Experiment 2 . Thus, in each experiment, the pattern of results differed between younger and older adults.

These age $\times$ duration interactions were confirmed in separate ANOVAs performed on the two experiments [Experiment $1, F(7,224)=6.68, p<.0001, \eta_{\mathrm{p}}^{2}=.17$; Experiment $\left.2, F(7,378)=3.70, p<.001, \eta_{\mathrm{p}}^{2}=.06\right]$. The effect of stimulus duration was also significant [Experiment 1, $F(7,224)=105.63, p<.0001, \eta_{\mathrm{p}}^{2}=.77$; Experiment 2 , $\left.F(7,378)=105.93, p<.0001, \eta_{\mathrm{p}}^{2}=.66\right]$. However, the age effect did not reach significance in Experiment 1 $\left[F(1,32)=1.53, p>.22, \eta_{\mathrm{p}}^{2}=.05\right]$ but was nearly significant in Experiment $2\left[F(1,54)=3.59, p<.07, \eta_{\mathrm{p}}^{2}=.06\right]$.

Figure 5 plots the probability that participants identified Tone $j$ as Tone $k,\left[p\left(R_{k} / S_{j}\right)\right]$ in Experiments 1 and 2. Figure 5 shows that, when Tone $j$ was presented, all younger adults identified it as Tone $j$ more often than they identified it as any other tone [i.e., $p\left(R_{j} / S_{j}\right)>p\left(R_{k} / S_{j}\right), k \neq j$ ].

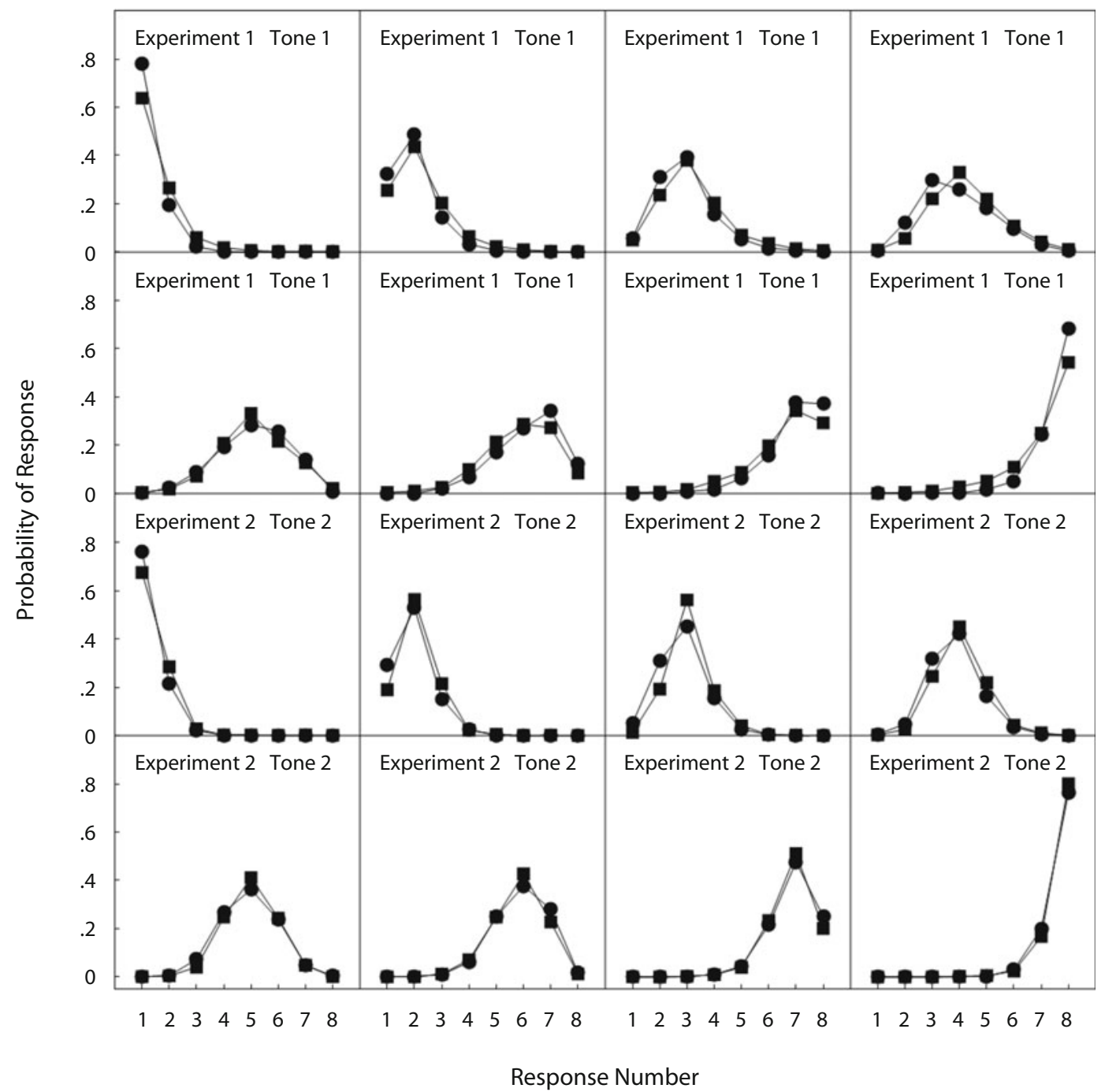

Figure 5. The probability that Response $k$ occurs when Stimulus $j$ is presented for each of the eight stimuli in Experiments 1 and 2 for young (squares) and old (circles) participants. 
Table 2

\begin{tabular}{|c|c|c|c|c|c|}
\hline \multirow{2}{*}{$\begin{array}{c}\text { Duration } \\
(\mathrm{msec})\end{array}$} & \multicolumn{2}{|c|}{$\begin{array}{l}\text { Location } \\
\text { of Mean }\end{array}$} & \multirow[b]{2}{*}{ Response } & \multicolumn{2}{|c|}{ Response Region } \\
\hline & Young & Old & & Young & Old \\
\hline \multicolumn{6}{|c|}{ Experiment 1} \\
\hline 220 & 0 & 0 & 1 & $x<0.22$ & $x<0.57$ \\
\hline 280 & 0.76 & 0.93 & 2 & $0.22<x<1.14$ & $0.57<x<1.73$ \\
\hline 355 & 1.57 & 1.97 & 3 & $1.14<x<1.90$ & $1.73<x<2.55$ \\
\hline 430 & 2.35 & 2.69 & 4 & $1.90<x<2.57$ & $2.55<x<3.14$ \\
\hline 520 & 2.93 & 3.57 & 5 & $2.57<x<3.15$ & $3.14<x<3.73$ \\
\hline 620 & 3.43 & 4.28 & 6 & $3.15<x<3.69$ & $3.73<x<4.35$ \\
\hline 730 & 3.96 & 4.96 & 7 & $3.69<x<4.40$ & $4.35<x<5.23$ \\
\hline 850 & 4.46 & 5.54 & 8 & $x>4.40$ & $x>5.23$ \\
\hline \multicolumn{6}{|c|}{ Experiment 2} \\
\hline 220 & 0 & 0 & 1 & $x<0.26$ & $x<0.51$ \\
\hline 300 & 0.96 & 0.93 & 2 & $0.26<x<1.46$ & $0.51<x<1.73$ \\
\hline 410 & 2.09 & 2 & 3 & $1.46<x<2.63$ & $1.73<x<2.82$ \\
\hline 560 & 3.09 & 3.05 & 4 & $2.63<x<3.54$ & $2.82<x<3.76$ \\
\hline 750 & 3.95 & 4.09 & 5 & $3.54<x<4.36$ & $3.76<x<4.56$ \\
\hline 1,000 & 4.69 & 4.96 & 6 & $4.36<x<5.24$ & $4.56<x<5.40$ \\
\hline 1,310 & 5.66 & 5.9 & 7 & $5.24<x<6.39$ & $5.40<x<6.51$ \\
\hline 1,700 & 7.03 & 7.03 & 8 & $x>6.39$ & $x>6.51$ \\
\hline
\end{tabular}

Figure 5 also shows that older adults in Experiment 1 sometimes identified Tone $j$ as Tone $k$ more often than they correctly identified it as Tone $j$ at the intermediate durations. In particular, they identified Tone 4 as Tone 3 more often than they identified it as Tone 4 , and Tone 6 as Tone 7 more often than they identified it as Tone 6 .

\section{A Signal Detection Analysis}

To determine whether response biases could account for the pattern of interactions shown in Figures 4 and 5, we performed a signal detection analysis of the data from Experiments 1 and 2, following the procedures described in Parker et al. (2002) (see Appendix B). As mentioned in the introduction, in these analyses, we also assumed that the distributions of events evoked along the decision axis were equal-variance Laplace distributions.

The signal detection analysis yielded the locations of the means of the distribution of response effects along a unidimensional decision axis for each of the stimuli in Experiments 1 and 2 and the locations of the criteria that define the response regions in these experiments (see Table 2). Figure 6 plots the locations of the stimuli along the identification decision axis as a function of stimulus duration for Experiment 1 (top panel) and Experiment 2 (bottom panel). From Figure 6, we can see that the identification values are a linear function of the logarithm of time for both younger and older adults in the two experiments $\left(r^{2} \geq .997\right.$ for all four functions). Moreover, the slope of the line relating projection values to the logarithm of time is steeper for older adults than it is for younger adults in both experiments. To determine whether this age-related difference in slopes was significant, we determined identification scale values for each of the 90 participants and plotted them against the logarithm of duration. The average value of $r^{2}$ for these plots was .99 for the young and old in Experiments 1 and 2.
A 2 (age) $\times 2$ (experiment) ANOVA on the logarithms of the slopes showed a significant effect of age $[F(1,86)=$ $\left.4.47, p<.05, \eta_{\mathrm{p}}^{2}=.05\right]$, but no significant effect of experiment $\left[F(1,86)=2.04, p>.15, \eta_{\mathrm{p}}^{2}=.02\right]$ and no significant age $\times$ experiment interaction $[F(1,86)=2.37, p>.10$, $\left.\eta_{\mathrm{p}}^{2}=.03\right]$. Hence, older adults performed better on the identification task than did younger adults.

In Figure 7, we plotted the identification scale values against the concatenated $d_{\mathrm{L}}^{\prime}$ scale values to examine the relationship between the identification and pairwise discrimination experiments. Figure 7 shows that the older adults appeared to have steeper linear functions in these coordinates than did younger adults. To determine whether this age-related difference in slopes was significant, we determined individual slopes for each of the 90 participants. A 2 (age) $\times 2$ (experiment) ANOVA on the logarithms of the slopes showed a significant effect of age $[F(1,86)=$ $\left.4.03, p<.05, \eta_{\mathrm{p}}^{2}=.045\right]$ and a significant effect of experiment $\left[F(1,86)=38.44, p<.0001, \eta_{\mathrm{p}}^{2}=.31\right]$, but no significant age $\times$ experiment interaction $[F(1,86)<1]$.

Note that a steeper slope in Figure 7 is associated with better performance relative to pairwise discriminability. Hence, compared with their pairwise discriminability, older adults performed significantly better than did younger adults.

\section{Other Models of Absolute \\ Identification of Duration}

In addition to fitting the Laplace signal detection model (described above) to the identification data, we also fit six other models: (1) a normal signal detection model; (2) a SET model; (3) the McCormack et al. (2002) memory model, without adjustments for response bias; (4) a single-parameter Laplace signal detection model; (5) the McCormack et al. (2002) memory model, with adjustments for response bias; 
and (6) an eight-parameter Laplace signal detection model. The procedure for fitting the normal signal detection model was identical to that used for fitting the Laplace signal detection model, except for the fact that equal-variance normal distributions were used instead of equal-variance Laplace distributions. In fitting the eight-parameter Laplace model, it was assumed that the locations of the stimuli along the decision axis were logarithmically related to stimulus duration (the same assumption as in the McCormack et al. [2002] model). The remaining eight parameters (the locations of the seven criteria and the $S D$ of the equal-variance Laplace distributions) were then fit following the usual procedure. In the one-parameter Laplace model, in addition to assuming that the locations of the stimuli along the decision axis were logarithmically related to stimulus duration, the locations of the seven criteria were set to midway between the means of the stimuli along the decision axis. This left only one param-

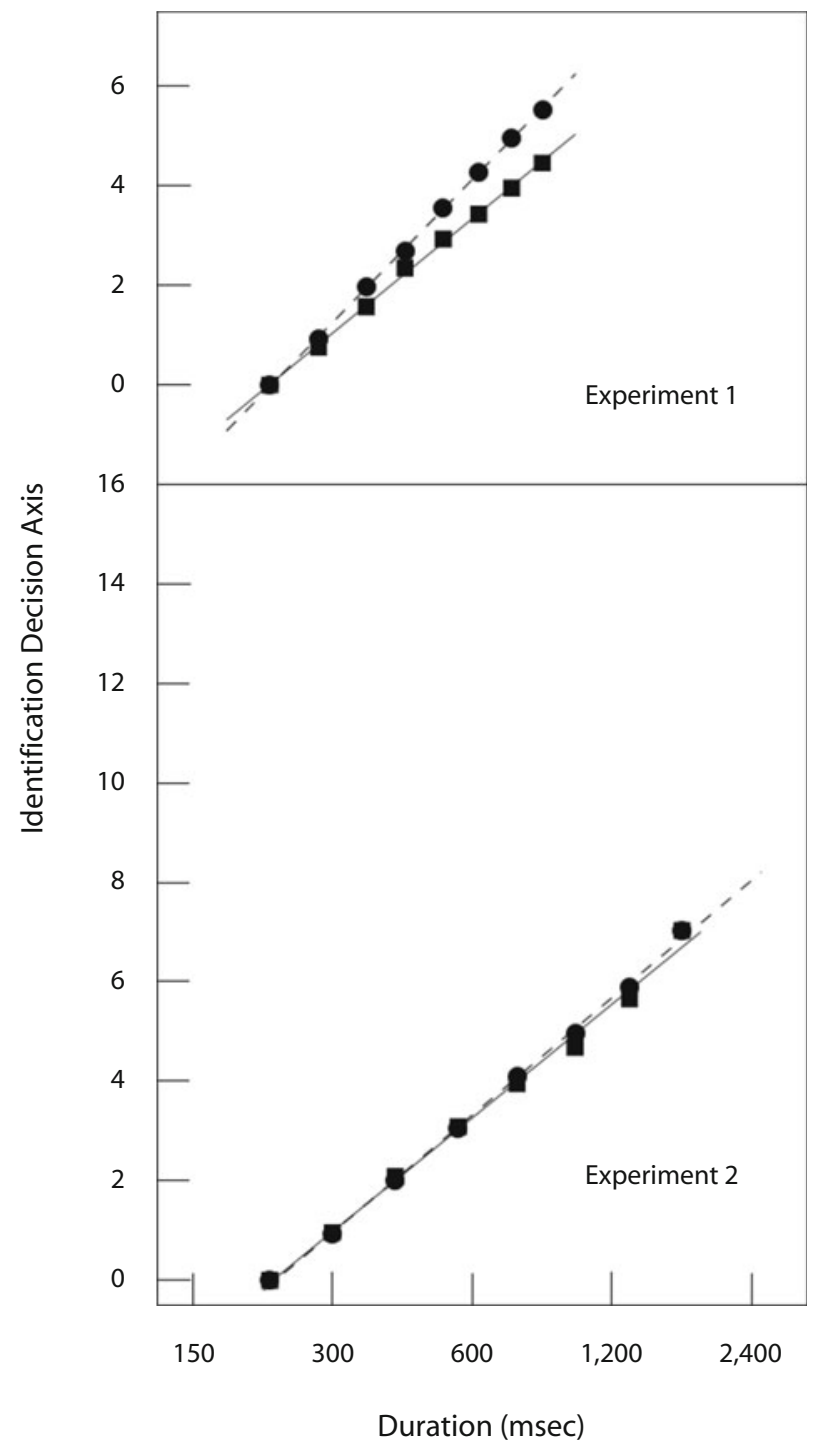

Figure 6. The locations of the stimuli along the identification decision axis as a function of stimulus duration for young (square) and old (circle) participants.

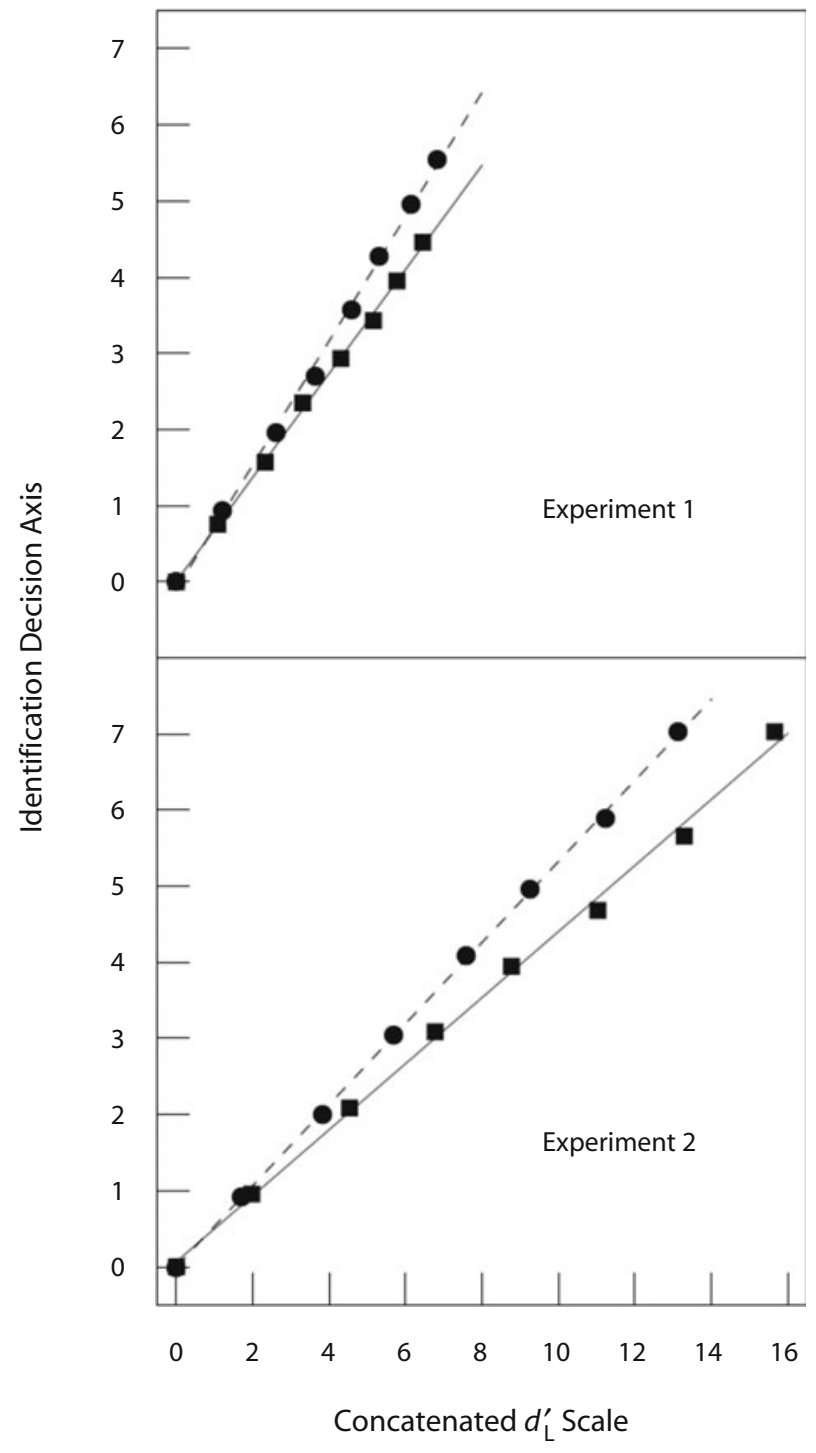

Figure 7. The locations of the stimuli along the identification decision axis as a function of their concatenated $d_{L}^{\prime}$ values for young (square) and old (circle) participants.

eter to fit-namely, the $S D$ of the equal-variance Laplace distributions. This parameter was determined using the same procedure for fitting all of the signal detection models. Details of how the SET models were fit can be found in Appendix A, and a description of how the remaining models were fit can be found in Appendix B.

Figure 8 plots the obtained probability of response as a function of the probabilities predicted by each of the models. It shows that the full Laplace signal detection model, LPfull, fitting seven means, seven criteria, plus the $S D$ of the Laplace distribution, provides the best fit to the data, followed by the eight-parameter Laplace signal detection model, LP8p, fitting seven criteria plus the $S D$ of the Laplace distribution. Interestingly, the one-parameter Laplace signal detection model, LP1p, provided a better fit than did the one-parameter SET model in all cases and a better fit than did the two-parameter McCormack et al. 


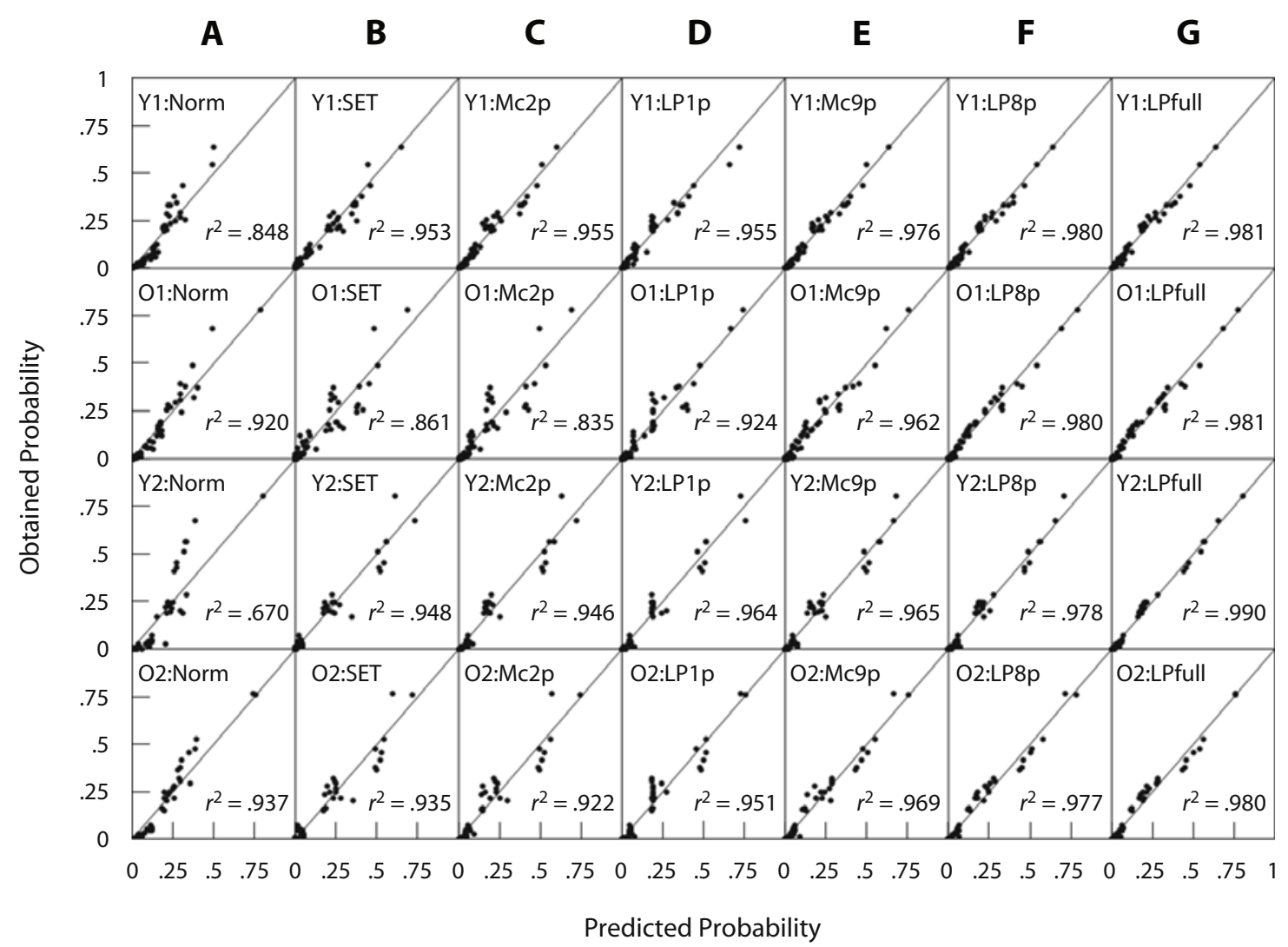

Figure 8. Obtained identification probabilities as a function of those predicted by one of seven models: (A) An equalvariance signal detection model with normal distributions of response effects (Norm); (B) a scalar expectancy theory model without a memory-distortion parameter (SET); (C) the McCormack et al. (2002) memory model without adjustment for response bias (Mc2p); (D) a single-parameter, equal-variance signal detection model with Laplace response distributions whose means are linearly related to log stimulus duration with criteria located midway between the means of the response distributions (LP1p); (E) the McCormack et al. (2002) memory model with adjustments for response bias (Mc9p); (F) the same model as LP1p, but with criterion locations as free parameters (LP8p); and (G) a full equalvariance Laplace signal detection model (LPfull).

(2002) model, Mc2p, in which no adjustments were made for response bias, in three of the four cases (the exception being the young adults in Experiment 1). Note also that the McCormack et al. (2002) nine-parameter model, $\mathrm{Mc} 9 \mathrm{p}$, which uses an additional seven parameters to adjust for response bias, performs almost as well as the eightparameter Laplace model, which also adjusts for response biases. Recall that, in both of these models, it is assumed that psychological duration is a logarithmic function of physical duration.

\section{DISCUSSION}

Figures 4 and 5 showed that the pattern of responding in the absolute identification experiments differed between younger and older adults. In both experiments, older adults tended to identify the anchor stimuli (the longest and shortest durations) more accurately than younger adults did but were poorer at identifying stimuli of intermediate duration than were younger adults. Second, when we examined the probability of identifying Tone $j$ as Tone $k(1 \leq k \leq 8)$, younger adults consistently identified Tone $j$ as Tone $j$ more frequently than they identified it as any other tone (see Figure 5). Older adults, on the other hand, sometimes identified Tone $j$ as Tone $j-1$ or Tone $j+1$ more frequently than they identified it as Tone $j$ (see Figure 5, Experiment 1). These age-related differences in the pattern of responding are quite similar to those found by McCormack et al. (2002), who found that older adults identified the intermediate durations less accurately than did younger adults and sometimes identified Tone $j$ as Tone $j-1$ more frequently than they identified it as Tone $j$, whereas younger adults consistently identified Tone $j$ as Tone $j$ more frequently than they identified it as any other tone. These age-related differences in the pattern of responding could be due to age-related differences in (1) discriminability, (2) response bias, (3) the representation of duration in memory, or (4) channel capacity (differences in the ability to identify unidimensional stimuli when the number of stimuli becomes large).

\section{Pairwise Discriminability}

The less accurate performance of older adults at the intermediate durations in the absolute identification ex- 
periments could have resulted from the fact that duration differences are less discriminable to them than to younger adults. To examine whether this factor could account, in part, for the age-related differences in performance, we determined the discriminability of adjacent pairs of stimuli in both younger and older adults. We found that the younger and older participants in both Experiments 1 and 2 did not differ statistically with respect to pairwise discriminability. Hence, age-related differences in pairwise discriminability are unlikely to contribute to agerelated differences in the pattern of responding.

\section{Response Bias or Memory Distortion?}

To evaluate whether the age-related differences in the patterns of responding were due to response biases or memory distortion, we fit three signal detection models and two memory-distortion models to the data from Experiments 1 and 2. Figure 8 shows that the full signal detection model, which assumes that events along the decision axis had a Laplace distribution, produced the best fit to the data. A plot of the means of these Laplace distributions against physical duration showed in all four cases that these means were related logarithmically to stimulus duration. Moreover, a reduced signal detection model, in which we assumed that the locations of the means of the distributions were related logarithmically to stimulus duration, fit the data almost as well as the full model. Hence, as in McCormack et al. (2002), the representation of the stimuli along the decision axis appears to be in terms of a logarithmic scale.

The equal-variance signal detection model, which assumed that events along the decision axis were distributed normally, provided the poorest fit to the data in three of the four cases. This suggests that events along the decision axis have a Laplace (exponential decay) distribution rather than a normal distribution. Hence, either the underlying distributions along the decision axis are Laplacian, or, as Schneider (2007) suggested, there is moment-to-moment (or person-to-person) variation in the $S D$ s of the normal distributions that generate the data.

It is also interesting to note that the McCormack et al. (2002) memory-distortion model assumes that the similarity between two stimuli is an exponential decay function of the subjective difference between them. Hence, the results of both the present experiment and those of McCormack et al. (2002) suggest that both the representation of events along the decision axis and the presumed similarity between them have distributions that fall off exponentially from their peak.

The SET model and the McCormack et al. (2002) memory model without an adjustment for response bias fared considerably more poorly than did either the full Laplace signal detection model or the eight-parameter reduced Laplace signal detection model. The eight-parameter Laplace model, however, employs seven parameters (the locations of the criteria in the $S D$ model) that allow for response biases. Hence, we also followed McCormack et al.'s (2002) procedure for adjusting the predictions of their two-parameter model, to ensure that the predicted probabilities of each response exactly matched the obtained probabilities of each response (see Appendix A). Because the obtained probabilities must sum to 1.0 , this adds seven parameters to the model. Figure 8 shows that adjusting for response bias in this fashion significantly improves the fit of the model to the data to the point at which it provides almost as good a fit as the eight-parameter Laplace $S D$ model. Hence, both models benefit substantially by taking into account the degree of response bias.

It is also interesting to note that, if response biases are controlled for in the McCormack et al. (2002) model, the discriminability parameter, $c$, does not differ substantially between younger and older adults. In their model, a large value of $c$ corresponds to a steep generalization gradient. It follows then that a steep generalization gradient should lead to greater discriminability between two stimuli. Indeed, in the McCormack et al. (2002) article and in the two experiments reported here, the value of $c$ was found to be higher for older than for younger adults ( 4.7 vs. 4.5 , 5.8 vs. 4.6 , and 4.8 vs. 4.7 , in McCormack et al. [2002], Experiment 1, and Experiment 2, respectively), suggesting that, if anything, older adults may be slightly better than younger adults with respect to the discriminability of stimuli in an identification experiment.

Another reason for thinking that the equal-variance Laplace model provides a better fit to the data is that the McCormack et al. (2002) memory model cannot easily account for the occasional instances in which Tone $j$ is identified as Tone $j-1$ or Tone $j+1$ more frequently than it is identified as Tone $j$. We show in Appendix A that if the tonal durations are logarithmically spaced (as they were in McCormack et al., 2002), and the parameters of the McCormack et al. (2002) model are adjusted so that an arbitrary Tone $j$ is identified as Tone $j-1$ more frequently than it is identified as Tone $j$, the McCormack et al. (2002) model predicts that this relationship will hold for all values of $j(j>1)$. Hence, if there is a distortion of this sort for one stimulus, all stimuli will be similarly distorted. Similarly, if the model predicts that an arbitrary Tone $j$ should be identified as Tone $j+1$ more frequently than it is identified as Tone $j$, then it predicts that this will happen for all values of $j(j<8)$. Both the McCormack et al. (2002) data and the present data show clear evidence that such distortions occur only for intermediate durations in older adults and that, in Experiment 1 of the present study, both types of distortion occur. Hence, the two-parameter McCormack et al. (2002) model (in which the predictions of the model are not adjusted so that the predicted response frequencies match the obtained response frequencies) is not sufficiently flexible to account for the kinds of response patterns observed both in their experiments and in ours. It is only when response biases are incorporated in the model that the model can fit cases in which Tone $j$ is identified as Tone $j-1$ more often than it is identified as Tone $j$ for some values of $j$, with the reverse being true for other values of $j$.

It is also interesting to compare a single-parameter SET theory with the single-parameter Laplace signal detection model. In SET theory, it is assumed that the primary source of variability comes from the memory component of the model (McCormack, Brown, Maylor, Darby, \& Green, 1999; McCormack et al., 2002; Wearden et al., 1997). If 
we assume, for the moment, that the clock count itself is variable and that the $S D$ of the clock count is proportional to the mean, then a logarithmic transformation of the clock counts associated with stimulus presentations would result in stimulus distributions along the decision axis whose variances would be essentially independent of stimulus duration. This version of SET theory then would predict that the mean locations of the stimuli along the decision axis should be proportional to the logarithm of their durations. The memory component in this model would be memory for the locations of the criteria that define the different response regions. This model has the advantage that performance is essentially independent of the degree of variation in the "remembered" positions of the criteria along the decision axis and depends only on their mean locations. These mean locations are essentially free to vary and could be affected by stimulus frequency, payoffs for correct and incorrect responses, and differences in response strategy. Hence, a slight recasting of SET theory in which the clock is variable with an $S D$ that is proportional to stimulus duration results in a signal detection model in which the mean locations of the stimuli along the decision axis are proportional to the logarithm of duration, with the $S D$ of each of these distributions approximately equal to one another.

Consider a specific example using the stimulus durations in Experiment 2. Assume a clock whose average count is directly proportional to stimulus duration. Assume further that there is some variability in the clock, so that the clock count to a stimulus whose duration is $T \mathrm{msec}$ is a normally distributed variable with a mean $=a * T$, and an $S D=$ $\tau * a * T$. If the participant makes a decision on the basis of the logarithm of the clock count, the distribution of events along the decision axis due to repeated presentations of a stimulus whose duration is $T$ msec would be a normally distributed variable whose mean is $\log [T]+\log [a]$ and whose $S D$ is independent of the value of $T$. Note that the temporal acuity of the individual is determined solely by the parameter $\tau$. That is, the smaller the value of $\tau$, the greater the separation between stimuli along the decision axis, and the better the discriminability.

According to this model, the distribution of events along the decision axis due to repeated presentations of a stimulus should be approximately normal if decisions are based on the logarithms of clock counts. However, here we found that Laplace distributions provided a better fit to the data than normal distributions (see Figure 8). Schneider (2007) has shown that if we average stimulus-response matrices across a set of individuals who have different sensitivities (in this example, different values of $\tau$ ), the average stimulus-response matrix is best fit by assuming that the underlying distributions along the decision axis are Laplacian rather than normal in shape. Hence, if the individuals in a study differ with respect to their sensitivity (have different values of $\tau$ ) or if the value of $\tau$ changes either systematically or randomly during or across sessions within an individual, then a Laplace rather than a normal model provides the best description of the decision process.

To check whether this also holds for decisions based on the logarithms of clock counts, we simulated a signal detection decision process in which decisions were based on the logarithm of clock counts when the sensitivity parameter $\tau$ itself was a random variable with a normal distribution, with $M=\mu_{\tau}$ and $S D=\sigma_{\tau}$. In this simulation, we used the eight durations from Experiment 2 and we set $a=1, \mu_{\tau}=$ .225 , and $\sigma_{\tau}=.0675$. This meant that the $S D$ of the clock for duration $T$ became $s * T$, where $s$ was a normal random variable whose mean was 0.225 and whose $S D$ was 0.0675 . Hence, when the presentation of a stimulus of duration $T$ was simulated, we randomly selected the value of $s$ from a normal distribution, with $M=0.225$ and $S D=0.0675$. We then generated a clock count $x$ from another normal distribution, whose mean was $T$ and whose $S D$ was $s * T$. The logarithm of $x$ then constituted an event along the decision axis, with the mean of the distribution of $x$ along the decision axis for a stimulus of $T$ msec being $\log [T]$. Because there were eight stimuli, this produced eight distributions of events along the decision axis. The locations of the seven criteria were set midway between the means of adjacent stimuli along the decision axis. In total, we simulated 100,000 presentations of each stimulus to determine the probability of response $i$, given stimulus $j$ for $1 \leq i \leq 8$ and $1 \leq j \leq 8$. We then found the best-fitting normal signal detection model and compared it with the one-parameter Laplace signal detection model. Figure 9 plots the obtained probabilities as a function of the predicted probabilities in both of these cases. Figure 9 shows that a single-parameter Laplace signal detection model is consistent with a clock model in which the $S D$ of the clock is proportional to stimulus duration and $\tau$ varies randomly across time or across participants. Given that the single-parameter Laplace model provides a very good fit to the data from these experiments, we conclude that the present results are completely compatible with an SET model in which the $S D$ of the clock is proportional to the duration of the stimulus, with the proportionality constant varying somewhat across participants or within and across sessions.

\section{Possible Bow Effects}

Identification performance is typically higher for "anchor" stimuli (e.g., the smallest and largest stimuli in the set) than it is for intermediate stimuli (the bow effect). Bow effects are explained sometimes in terms of sensory processes (e.g., Braida \& Durlach, 1972) and sometimes in terms of decision processes (e.g., Lacouture, Grondin, \& Mori, 2001). Hence, it is possible that the age-related differences in response patterns observed in these experiments reflect age-dependent bow effects (Figure 4 suggests that the bow effect, if it exists, might be greater for older than for younger adults). According to Braida and Durlach, the variance in the decision variable increases with the distance that one is from the anchor stimuli (the smallest and largest stimuli in the set). This would result in better discriminability between Stimuli 1 and 2 and between Stimuli 7 and 8 than between intermediate stimuli pairs (see also Braida et al., 1984). Note that, within the SDT model, a bow or anchor effect would imply that the variance along the decision axis is not uniform (variances would be larger for stimuli in the middle). As Figure 8 shows, however, an equal-variance Laplace model fits the data very well for both younger and older participants. If older participants 


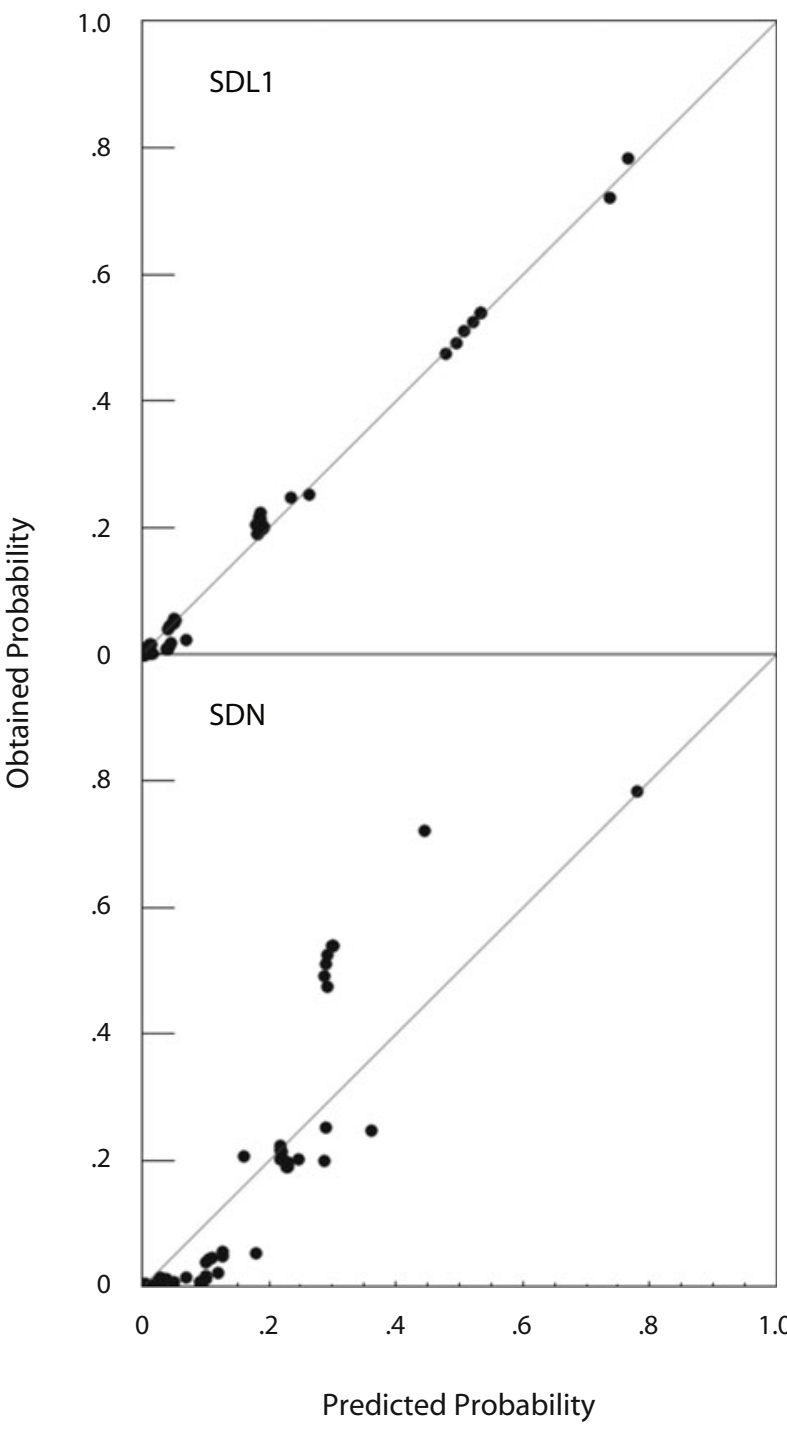

Figure 9. Simulated identification probabilities as a function of those predicted by a single-parameter, signal detection model with Laplace response distributions (SDL1, top panel) and by the full equal-variance, normal-distribution, signal detection model (SDN, bottom panel). The simulated identification probabilities were based on a single-parameter scalar expectancy theory model, in which the response distributions were normal in shape for each individual but the $S D$ of these response distributions were allowed to vary from one individual to the next (see text).

were better than younger participants at using the anchors, we would expect a poorer fit for older than for younger listeners. As Figure 8 shows, there is no evidence that this is the case. Moreover, when we relaxed the equal-variance assumption for the group data, there was very little improvement in fit in either younger or older adults. The addition of seven parameters (the $S D$ of Stimulus 2 was fixed at 1.0, and the other seven $S D$ s were allowed to vary) reduced normalized $\chi^{2}$ by only $21 \%$ for the younger adults in Experiments 1 and 2 and by $7 \%$ and $5 \%$ for the older adults in Experiments 1 and 2, respectively. Hence, a 50\% increase in the number of fitted parameters produced only a marginal improvement in fit. Moreover, there was no in- dication that the $S D$ associated with a stimulus distribution varied systematically with the position of the stimulus (whether it was at the edge of the range or in the middle). Hence, it is unlikely that the better performance of older adults in the identification experiments was due the bow effect being greater for them than for young adults.

\section{Age-Related Difference in Channel Capacity}

It has long been known that unidimensional sensory channels are limited in terms of the amount of information they can transmit (channel capacity; Miller, 1956). Hence, it is reasonable to ask whether channel capacity decreases with age. In the present set of experiments, we measured absolute identification accuracy when only two stimuli were presented during the identification task and when eight stimuli were presented during the identification task. Given limitations on channel capacity and the tendency for the variance in the signal detection model to increase with increases in the number of stimuli and/or in stimulus range (e.g., memory variance; Durlach \& Braida, 1969), we would expect identification accuracy to be highest when only two stimuli were presented. Because we tested all adjacent pairs of stimuli in the two-alternative absolute identification task, we were able to construct, assuming additivity among adjacent $d_{\mathrm{L}}^{\prime} \mathrm{s}$, the decision axis that we would expect in the eight-alternative absolute identification task, assuming that there was no diminution of discrimination accuracy for eight as opposed to two stimuli. In Figures 3 and 7, this hypothetical decision axis is referred to as the concatenated $d_{\mathrm{L}}^{\prime}$ scale.

If an unlimited amount of duration information could be transmitted, we would expect that the decision axis in the eight-alternative identification experiment would be the same as the concatenated $d_{\mathrm{L}}^{\prime}$ scale and that, if we plotted one against the other, we would find a linear relation with a slope equal to 1 . However, if channel capacity was limited in some way, we might still expect to find a linear relationship, but with a slope less than 1 . Plots of the relationship between the identification decision axis and the concatenated $d_{\mathrm{L}}^{\prime}$ scales (see Figure 7) show that the relationship is indeed linear, with slopes less than 1 for the young and old participants in Experiments 1 and 2, indicating that the amount of information that can be transmitted, relative to pairwise discriminability, is limited when multiple stimuli are involved. It is interesting to note that this limitation is more severe for younger than for older adults. In other words, older adults are better than younger adults at identifying stimuli varying only in duration, once we take into account the ability of each age group to distinguish one duration from another. Hence, relative to their baseline discriminability, older adults appear to have a larger channel capacity than younger adults do.

Although Murphy et al. (2006) found no age-related differences for absolute judgments of tonal intensity, McCormack et al. (2002) came to the opposite conclusionnamely, that older adults perform more poorly than do younger adults in absolute identification of auditory temporal duration. As we have seen, this conclusion, in part, was made on the basis of the evidence from a two-group (young vs. old) $\times$ nine-stimuli ANOVA, since the fitted 


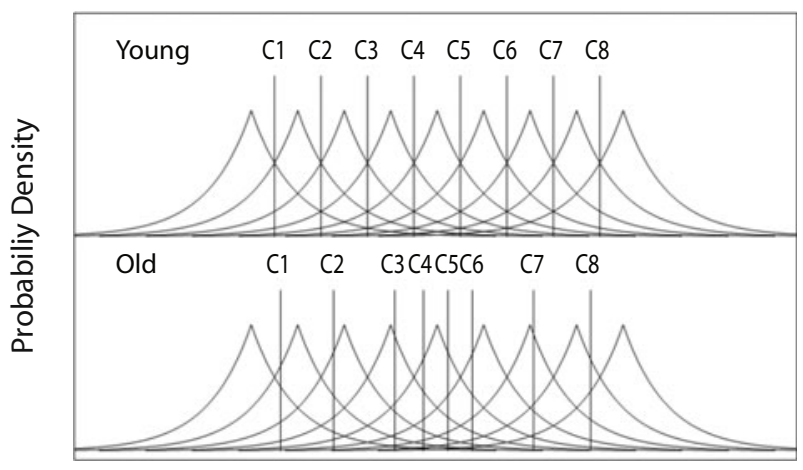

Decision Axis

Figure 10. Hypothetical distributions for younger and older adults in an signal detection theory model, in which the means of the response distributions are equal to the logarithms of the stimulus durations in Experiment 2 of McCormack et al. (2002) and in which the $S D$ s of these response distributions are the same for all stimuli and for both younger and older adults. In this model, the only differences between younger and older adults are the locations of the criteria.

value of the generalization parameter would indicate that older adults had generalization gradients at least as steep as those of younger adults. This, in turn, suggests that the age-related differences found in the ANOVAs of the present experiment and those of McCormack et al. (2002) may be due primarily to response biases. To see whether response biases could produce the pattern of ANOVA results found here and in McCormack et al. (2002) when there were no age-related differences in discriminability in the identification experiment, we simulated 1,000 experiments (using the stimulus durations from Experiment 2 of McCormack et al., 2002), in which we assumed that the locations of the midpoints of the distribution of effects due to a stimulus along the decision axis were given by the common logarithm of the stimulus durations for both younger and older adults. In addition, we assumed that all distributions were Laplacian in form and had the same $S D$ for both younger and older adults. The only difference between the assumed decision process in younger and older adults was that they differed with respect to criterion location, as indicated in Figure 10. Note that, in this formulation, the degree of discriminability among stimuli is completely governed by the $S D, \sigma_{\mathrm{L}}$, of the Laplace distributions. This was assumed to be constant for an individual participant but to vary randomly across participants. However, the distribution of this random variable was assumed to be the same in both younger and older adults $\left(\sigma_{\mathrm{L}}\right.$ was normally distributed, with $M=.15$ and $S D=.02$ in both populations). An experiment consisted of a random selection of 21 younger and older participants from this hypothetical pool, in which the distribution of $\tau$ was identical for both age groups. Each hypothetical participant was presented with each stimulus 15 times, and the number of times each stimulus was identified correctly was recorded. The only difference between the two age groups was in the placement of the criteria along the decision axis. We then conducted a 2 (age) $\times 9$ (stimulus) ANOVA on the data and tested for significant effects due to age, to stimulus, and to an interaction between age and stimulus. Assuming an $\alpha$ value (probability of a Type I error) of .05, we found a significant age effect in 830 of the 1,000 simulations and a significant stimulus effect and age $\times$ stimulus interaction in all 1,000 simulations, with older adults having poorer identification performance, on average, than younger adults. Hence, response bias and discriminability effects are confounded if one conducts an ANOVA only on correct responses. Therefore, the most parsimonious interpretation of the age effects found in the McCormack et al. (2002) study is that they reflect age-related differences in response strategies or response bias.

\section{Implications for SET}

If the present results are interpreted with respect to a clock model, the absence of age-related differences for the pairwise comparisons would be consistent with the average values of $\mu_{\tau}$ and $\sigma_{\tau}, M$ and $S D$ of $\tau$ in the SET model being approximately the same for younger and older adults. Note that increases in either $\mu_{\tau}$ or $\sigma_{\tau}$, as the number of stimuli in an absolute identification experiment increases, would result in poorer discriminability. Hence, the decrease in discriminability that occurs with increases in the number of stimuli could be due to increases in either $\mu_{\tau}$ or $\sigma_{\tau}$. Moreover, if either parameter increased with increases in the number of stimuli at a slower rate for older than for younger adults, there would be a proportionately greater loss of sensitivity in younger than in older adults, as was found in these experiments.

In terms of the Durlach and Braida (1969) model, the present results suggest that, as the number of stimuli in an identification experiment increases, memory variance actually grows at a slower rate for older than for younger adults. Specifically, if we assume that the locations of the mean responses along the decision axis are the same for younger and older adults, as suggested by the fact that we found no age-related differences in pairwise discriminability in the two experiments, then the sum of stimulus variance and memory variance, when two adjacent stimuli are presented, has to be the same for younger and older adults. Now, if we assume that stimulus variance does not change when additional stimuli are presented in the absolute identification paradigm, any loss in discriminability has to be due to an increase in memory variance. Because older adults perform better than younger adults, relative to their ability to discriminate adjacent pairs of stimuli, the growth in memory variance when the stimulus set is increased from two to eight stimuli must be less for older than for younger adults. At the very least, it suggests that, as the number of stimuli in an identification experiment increases, memory variance does not grow at a faster rate for older than it does for younger adults and that the channel capacity for duration is not reduced by age.

In conclusion, the results of the present study suggest that age-related differences in the pattern of responding in an absolute identification experiment reflect age-related differences in response bias rather than age-related differences in channel capacity. Moreover, these results suggest that older adults can process a large number of 
unidimensional stimuli varying only in duration at least as well as younger adults can. Moreover, there are indications in the data that, relative to the ability of each group to discriminate between two such stimuli, older adults may be somewhat better than younger adults in an identification task. Additional experiments are needed to determine whether the capacity to handle a large number of stimuli is undiminished, and is perhaps even enhanced, with age in stimulus dimensions other than intensity and time.

\section{AUTHOR NOTE}

A portion of this research was presented at the 111th American Psychological Association Convention in August, 2005. This research was supported by a grant from the Natural Sciences and Engineering Research Council of Canada. We thank Michael Palangio and Kelly Burkholder for assistance in conducting these experiments. H.B. is now at the Department of Psychology, Ryerson University, Toronto. Address correspondence to D. R. Murphy, Department of Psychology, Nipissing University, 100 College Dr., North Bay, ON, P1B 8L7 Canada (e-mail: danam@nipissingu.ca).

\section{REFERENCES}

Allan, L. G. (1998). The influence of the scalar timing model on human timing research. Behavioural Processes, 44, 101-117. doi:10.1016/ S0376-6357(98)00043-6

Bergeson, T. R., Schneider, B. A., \& Hamstra, S. J. (2001). Duration discrimination in younger and older adults. Canadian Acoustics, 29, 3-10.

Bilger, R. C., Nuetzel, M. J., Rabinowitz, W. M., \& Rzeczkowski, C. (1984). Standardization of a test of speech perception in noise. Journal of Speech \& Hearing Research, 27, 32-48.

Block, R. A., ZaKaY, D., \& HanCOCK, P. A. (1998). Human aging and duration judgments: A meta-analytic review. Psychology \& Aging, 13, 584-596. doi:10.1037/0882-7974.13.4.584

BraIDA, L. D., \& DURLACH, N. I. (1972). Intensity perception: II. Resolution in one-interval paradigms. Journal of the Acoustical Society of America, 51, 483-502. doi:10.1121/1.1912868

Braida, L. D., Lim, J. S., Berliner, J. E., Durlach, N. I., RabinoWITZ, W. M., \& PURKS, S. R. (1984). Intensity perception: XIII. Perceptual anchor model of context-coding. Journal of the Acoustical Society of America, 76, 722-731. doi:10.1121/1.391258

Brown, G. D. A., McCormack, T., Smith, M., \& Stewart, N. (2005). Identification and bisection of temporal durations and tone frequencies: Common models for temporal and nontemporal stimuli. Journal of Experimental Psychology: Human Perception \& Performance, 31, 919-938. doi:10.1037/0096-1523.31.5.919

Brownell, W. E. (1997). How the ear works: Nature's solutions for listening. Volta Review, 99, 9-28.

Craik, F. I. M., \& HAY, J. F. (1999). Aging and judgments of duration: Effects of task complexity and method of estimation. Perception \& Psychophysics, 61, 549-560.

Durlach, N. I., \& BRAIDA, L. D. (1969). Intensity perception: I. Preliminary theory of intensity resolution. Journal of the Acoustical Society of America, 46, 372-383. doi:10.1121/1.1911699

Gordon, M. S., \& SchneIder, B. A. (2007). Gain control in the auditory system: Absolute identification of intensity within and across two ears. Perception \& Psychophysics, 69, 232-240.

Ison, J. R., Virag, T. M., Allen, P. D., \& HAmmond, G. R. (2002). The attention filter for tones in noise has the same shape and effective bandwidth in the elderly as it has in younger listeners. Journal of the Acoustical Society of America, 112, 238-246. doi:10.1121/1.1483321

Lacouture, Y., Grondin, S., \& Mori, S. (2001). Absolute identification of temporal intervals: Preliminary data. In G. Sommerfeld, R. Kompass, \& T. Lachmann (Eds.), Fechner Day 2001: Proceedings of the 17th Annual Meeting of the International Society for Psychophysics (pp. 493-498). Lengerich, Germany: Pabst.

LEVITT, H. (1971). Transformed up-down methods in psychoacoustics. Journal of the Acoustical Society of America, 49, 467-477. doi:10.1121/ 1.1912375
Macmillan, N. A., \& Creelman, C. D. (2005). Detection theory: A user's guide (2nd ed.). Mahwah, NJ: Erlbaum.

McCormack, T., Brown, G. D. A., Maylor, E. A., Darby, R. J., \& GreEN, D. (1999). Developmental changes in time estimation: Comparing childhood and old age. Developmental Psychology, 35, 11431155. doi:10.1037/0012-1649.35.4.1143

McCormack, T., Brown, G. D. A., Maylor, E. A., Richardson, L. B. N., \& DARBY, R. J. (2002). Effects of aging on absolute identification of duration. Psychology \& Aging, 17, 363-378. doi:10.1037/0882 $-7974.17 .3 .363$

Meck, W. H. (1996). Neuropharmacology of timing and time perception. Cognitive Brain Research, 3, 227-242. doi:10.1016/0926 $-6410(96) 00009-2$

Miller, G. A. (1956). The magical number seven, plus or minus two: Some limits on our capacity for processing information. Psychological Review, 63, 81-97. doi:10.1037/h0043158

Murphy, D. R., Schneider, B. A., Speranza, F., \& Moraglia, G. (2006). A comparison of higher-order auditory processes in younger and older adults. Psychology \& Aging, 21, 763-773. doi:10.1037/0882 $-7974.21 .4 .763$

Parker, S., Murphy, D. R., \& Schneider, B. A. (2002). Top-down gain control in the auditory system: Evidence from identification and discrimination experiments. Perception \& Psychophysics, 64, 598-615.

Perbal, S., Droit-Volet, S., Isingrini, M., \& Pouthas, V. (2002). Relationships between age-related changes in time estimation and agerelated changes in processing speed, attention, and memory. Aging, Neuropsychology, \& Cognition, 9, 201-216. doi:10.1076/anec.9.3 201.9609

Pichora-Fuller, M. K., Schneider, B. A., \& Daneman, M. (1995). How young and old adults listen to and remember speech in noise. Journal of the Acoustical Society of America, 97, 593-608. doi: $10.1121 / 1.412282$

Robles, L., \& Ruggero, M. A. (2001). Mechanics of the mammalian cochlea. Physiological Reviews, 81, 1305-1352.

SchneIDER, B. A. (1997). Psychoacoustics and aging: Implications for everyday listening. Journal of Speech-Language Pathology \& Audiology, 21, 111-124.

SCHNEIDER, B. A. (2007). The shape of the underlying distributions in absolute identification experiments. In S. Mori \& W. Wong (Eds.), Fechner Day 2007: Proceedings of the 23rd Annual Meeting of the International Society for Psychophysics (pp. 171-176). Tokyo: International Society for Psychophysics.

Schneider, B. A., \& Pichora-Fuller, M. K. (2000). Implications of perceptual deficits for cognitive aging research. In F. I. M. Craik \& T. A. Salthouse (Eds.), The handbook of aging and cognition (2nd ed., pp. 155-219). Mahwah, NJ: Erlbaum.

Schneider, B. A., \& Pichora-Fuller, M. K. (2001). Age-related changes in temporal processing: Implications for speech perception. Seminars in Hearing, 22, 227-239.

Schneider, B. A., Pichora-Fuller, M. K., \& Daneman, M. (2009). Effects of senescent changes in audition and cognition on spoken language comprehension. In S. Gordon-Salant, R. D. Frisina, A. N. Popper, \& R. R. Fay (Eds.), The aging auditory system (Springer Handbook of Auditory Research, Vol. 34, pp. 167-210). New York: Springer.

Sheldon, S., Pichora-Fuller, M. K., \& Schneider, B. A. (2008). Priming and sentence context support listening to noise-vocoded speech by younger and older adults. Journal of the Acoustical Society of America, 123, 489-499. doi:10.1121/1.2783762

WeArden, J. H. (2004). Decision processes in models of timing. Acta Neurobiologiae Experimentalis, 64, 303-317.

Wearden, J. H., Wearden A. J., \& Rabbitt, P. M. A. (1997). Age and IQ effects on stimulus and response timing. Journal of Experimental Psychology: Human Perception \& Performance, 23, 962-979. doi:10.1037/0096-1523.23.4.962

YATES, G. K. (1995). Cochlear structure and function. In B. C. J. Moore (Ed.), Hearing: Handbook of perception and cognition (2nd ed., pp. 41-74). San Diego: Academic Press.

\section{NOTE}

1. Because the distribution of these slopes was positively skewed and the $S D$ of these distributions increased with the mean, the slopes were $\log$ transformed. 


\section{APPENDIX A}

\section{Scalar Expectancy Theories}

\section{The McCormack Model}

According to the McCormack et al. (2002) memory model of the absolute identification experiment, the psychological similarity between stimulus duration $S_{i}$ and stimulus duration $S_{j}$ is $\eta_{i, j}=e^{-c\left|M_{i}-M_{j}\right| \text {, where } M_{j}}$ is the psychological representation of stimulus duration $S_{j}$, and $c>0$ is a parameter of the model. The model further supposes that the representation of $S_{k}$ in memory is $M_{k}=\log _{e}\left(S_{k}\right)+D$, where $D$ represents a distortion of duration in memory and is the second parameter of the model. Note that, on trials in which stimulus duration $S_{i}$ is presented, its psychological representation is $M_{i}=\log _{e}\left(S_{i}\right)$ (there is no distortion, because the stimulus is physically present). Hence, on trials in which physical duration $S_{i}$ is presented, the similarity between physically presented $S_{i}$ and remembered $S_{j}$ is

$$
\eta_{i, j}=e^{-c\left|\log _{e}\left(S_{i}\right)-\log _{e}\left(S_{j}\right)-D\right|}=e^{-c\left|\log _{e}\left(\frac{S_{i}}{S_{j}}\right)-D\right|} .
$$

Now, the model also states that the probability of response $j$ to physically presented $S_{i}$ is

$$
p\left(R_{j} / S_{i}\right)=\frac{\eta_{i, j}}{\sum_{k=1}^{n} \eta_{i, k}}=\frac{e^{-c\left|\log _{e}\left(\frac{S_{i}}{S_{j}}\right)-D\right|}}{\sum_{k=1}^{n} e^{-c\left|\log _{e}\left(\frac{S_{i}}{S_{k}}\right)-D\right|}} .
$$

\section{Fitting the McCormack Model}

To fit the model to the present data, we computed the Pearson $\chi^{2}$ statistic

$$
\chi^{2}=\sum_{i=1}^{n} \sum_{j=1}^{n} \frac{\left[N\left(R_{j} / S_{i}\right)-N_{s} p\left(R_{j} / S_{i}\right)\right]^{2}}{N_{s} p\left(R_{j} / S_{i}\right)},
$$

where $N\left(R_{j} / S_{i}\right)$ is the number of times duration $S_{i}$ was labeled as response $R_{j}$, and $N_{s}$ is the number of times that each duration was presented during the experiment. We then varied the $c$ and $D$ parameters in a systematic fashion to find a minimum value for $\chi^{2}$. This was done first without employing their procedure for adjusting response bias. Hence, without the response bias adjustment, there are only two parameters in the model.

\section{Adjusting for Response Bias in the McCormack Model}

To adjust for response biases, McCormack et al. (2002) employed an iterative procedure to adjust the predictions of the two-parameter model until the predicted frequencies of the eight responses matched the frequencies obtained in the data. They took the predicted stimulus-response matrix, in which rows were stimuli, columns were responses, and entries were the probability of response $j$ given stimulus $i$, and iteratively adjusted it as follows. They summed each column in the obtained stimulus-response matrix and each column in the predicted stimulus-response matrix. They then divided each entry in a column in the predicted stimulus-response matrix by the column total in the predicted stimulus-response matrix and then multiplied the resultant entries in that column by the corresponding column total in the obtained stimulus-response matrix. These operations produced predicted response frequencies that matched obtained response frequencies, but they had the effect of altering the row totals in the predicted stimulus-response matrix so that they no longer added up to 1.0. They corrected this by dividing the entries in a row of the adjusted prediction matrix by the row totals in that matrix. They repeated this sequence of operations until the adjusted prediction matrix had row totals summing to 1.0 and column totals equal to those in the obtained stimulus-response matrix. Hence, this model forced the column totals in the prediction matrix to equal those in the obtained matrix. In fitting this model, we iteratively searched different values of $c$ and $D$, adjusted the predicted response matrix produced by these values of $c$ and $D$ until their column totals in the predicted stimulus-response matrix equaled the column totals in the obtained data matrix, and then computed the $\chi^{2}$ goodness-of-fit measure. Hence, this is a nine-parameter model, since it fits $c$ and $D$ as well as eight column totals (seven degrees of freedom in the column totals).

\section{Memory Biases in the McCormack Model}

A major feature of the McCormack et al. (2002) model was that it was supposed to account for the fact that, for older adults, it was sometimes the case that $p\left(R_{i-1} \mid S_{i}\right)>p\left(R_{i} \mid S_{i}\right)$. To see what this implies in the model, we examine the ratio of $p\left(R_{i-1} \mid S_{i}\right)$ to $p\left(R_{i} \mid S_{i}\right)$ :

$$
p\left(R_{i-1} \mid S_{i}\right)=\frac{e^{-c\left|\log _{e}\left(\frac{S_{i}}{S_{i-1}}\right)-D\right|}}{\sum_{k=1}^{n} e^{-c\left|\log _{e}\left(\frac{S_{i}}{S_{k}}\right)-D\right|}}, p\left(R_{i} \mid S_{i}\right)=\frac{e^{-c\left|\log _{e}\left(\frac{S_{i}}{S_{i}}\right)-D\right|}}{\sum_{k=1}^{n} e^{-c\left|\log _{e}\left(\frac{S_{i}}{S_{k}}\right)-D\right|}}, \frac{p\left(R_{i-1} \mid S_{i}\right)}{p\left(R_{i} \mid S_{i}\right)}=\frac{e^{-c\left|\log _{e}\left(\frac{S_{i}}{S_{i-1}}\right)-D\right|}}{e^{-c|-D|}} .
$$


APPENDIX A (Continued)

Assuming that the ratio of these two probabilities is 1.0 for $1<i \leq n$, we would have

$$
e^{-c\left|\log _{e}\left(\frac{S_{i}}{S_{i-1}}\right)-D\right|}=e^{-c|-D|} .
$$

Note that this can be true only if

$$
\left|\log _{e}\left(\frac{S_{i}}{S_{i-1}}\right)-D\right|=|-D| .
$$

Now, note that the ratio of $S_{i}$ to $S_{i-1}$ is always constant and greater than 1 in McCormack's experiments. Let

$$
r=\log _{e}\left(\frac{S_{i}}{S_{i-1}}\right), r>0 .
$$

Hence, in order for the ratio of the $p\left(R_{i-1} \mid S_{i}\right)$ to $p\left(R_{i} \mid S_{i}\right)=1,|r-D|=|-D|$. Now, when $D<0$, it is obvious that $|r-D|>|-D|$ for $D<0$. Hence, the probability of $p\left(R_{i-1} \mid S_{i}\right)$ to $p\left(R_{i} \mid S_{i}\right)$ cannot be equal to 1 when $D<0$, nor can it be greater than 1 if $D<0$.

Now, if $D>0,|r-D|=|-D|$ iff $D=r / 2$, and $|r-D|<|-D|$ for $D>r / 2$. Since

then

$$
\frac{p\left(R_{i-1} \mid S_{i}\right)}{p\left(R_{i} \mid S_{i}\right)}=\frac{e^{-c|r-D|}}{e^{-c|-D|}}=e^{-c(|r-D|-|-D|)},
$$

$$
\frac{p\left(R_{i-1} \mid S_{i}\right)}{p\left(R_{i} \mid S_{i}\right)}>1
$$

if and only if $D>r / 2$. Hence, $D$ must be greater than $r / 2$ in order for the ratio of $p\left(R_{i-1} \mid S_{i}\right)$ to $p\left(R_{i} \mid S_{i}\right)$ to be greater than 1. Please note another feature of this model. If $D>r / 2$, the ratio of $p\left(R_{i-1} \mid S_{i}\right)$ to $p\left(R_{i} \mid S_{i}\right)$ is greater than 1 for all $1<i \leq n$. Therefore, according to these derivations, the model proposed by McCormack et al. (2002) cannot provide a good fit to data for which there are only a limited number of cases in which the ratio of $p\left(R_{i-1} \mid S_{i}\right)$ to $p\left(R_{i} \mid S_{i}\right)$ is greater than 1, because, according to the model, if it holds for any stimulus $(i>1)$, it holds for all stimuli $(i>1)$. However, if the procedure for adjusting for response bias is applied, this prediction of the model can be overridden.

\section{A Single-Parameter SET Model}

In another version of SET theory (e.g., Wearden, 2004), the listener compares the clock count elicited by a stimulus of duration $t$ with the remembered clock count $m_{j}$ for each of the $j$ stimuli- that is, for an interval of length $t$, the observer computes

$$
\frac{\operatorname{Abs}\left(t-m_{1}\right)}{t}, \frac{\operatorname{Abs}\left(t-m_{2}\right)}{t}, \ldots, \frac{\operatorname{Abs}\left(t-m_{8}\right)}{t} .
$$

The observer then identifies the interval with the decision variable having the lowest value. Because the $m \mathrm{~s}$ are random variables, sometimes the interval $t$ is misidentified. This decision rule then generates an $8 \times 8$ stimulusresponse matrix. Note that the probabilities in this response matrix depend only on the value of $c$, where the $S D$ of the normal distribution of $m$ is assumed to be equal to $c * t$. To find the predicted probabilities for a particular value of $c$, we simulated 100,000 trials for each stimulus presentation. We then looked for the value of $c$ that produced the smallest sum of squared differences between predicted and obtained probabilities. (Note that we did not minimize $\chi^{2}$ in this instance, because, even with 100,000 trials, the Monte Carlo estimates of the probabilities of some of the responses to a stimulus were 0 , which would yield an infinite $\chi^{2}$ value.) The best-fitting values of $c$ were 0.21 and 0.22 for the young observers in Experiments 1 and 2, respectively, and 0.19 and 0.23 for the old observers in Experiments 1 and 2, respectively. 


\section{APPENDIX B \\ Signal Detection Models}

In a signal detection analysis of an absolute identification experiment, each of $n$ stimuli is assumed to give rise to a distribution of effects along a decision axis. Furthermore, the observer is assumed to divide the decision axis into $n$ response regions. Figure B1 illustrates this process for $n=8$. Here, each of eight stimuli gives rise to eight distributions, with all distributions having the same variance and shape (Laplace).

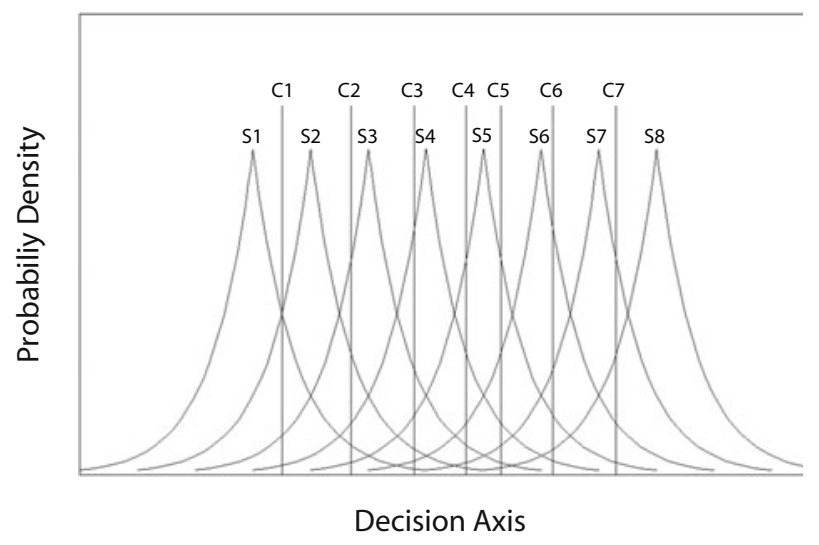

Figure B1. Hypothetical distributions of responses elicited by the presentation of one of any eight stimuli (S1-S8). All distributions have the same variance and shape (Laplace). The mean of the first distribution is labeled "S1," and its corresponding response region (RR1) is that portion of the decision axis to the left of Criterion $1(C 1)$; RR2 is the portion of the decision axis between $\mathrm{C} 1$ and $\mathrm{C} 2, \mathrm{RR} 3$ is the portion of the decision axis between $\mathrm{C} 2$ and $\mathrm{C} 3, \ldots$, and $\mathrm{RR8}$ is the portion of the decision axis to the right of $\mathrm{C} 7$.

The data from the experiment consists of an $n \times n$ stimulus-response matrix, in which the entries are the number of times stimulus $i$ was identified as stimulus $j$. When these entries are divided by the total number of times that each stimulus was presented in the experiment $\left(N_{S}\right)$, the entries become $p_{o}\left(R_{j} \mid S_{i}\right)$, which we refer to as the obtained probability of response $j$, given stimulus $i$. The probabilities predicted by the model, $p\left(R_{j} \mid S_{i}\right)$, are obtained by integrating the distribution evoked by stimulus $i$ over response region $j$. Now, if we fix the mean of one of the distributions at 0 and their $S D$ s at 1.0, there are 14 free parameters in the model, the means of the distributions for Stimuli $2-8$, and the locations of the seven criteria along the decision axis. In the general case, in which there are $n$ stimuli, there are $2(n-1)$ fitted parameters.

The fitting procedure determined the values of these 14 parameters that minimized norm $\chi^{2}$, where

$$
\operatorname{norm} \chi^{2}=\sum_{j=1}^{n} \sum_{i=1}^{n} \frac{\left[p_{o}\left(R_{j} \mid S_{i}\right)-p\left(R_{j} \mid S_{i}\right)\right]^{2}}{p\left(R_{j} \mid S_{i}\right)},
$$

using the method described in Parker et al. (2002). Note that, when norm $\chi^{2}$ is multiplied by $N_{S}$, it becomes a $\chi^{2}$ variable with $n^{2}-3 n+2$ degrees of freedom. Hence, the fitting procedure effectively minimized $\chi^{2}$.

In addition to the full Laplace model described above, we also fit five other models to our obtained data. In the first of these, the distributions along the decision axis were assumed to be normal and of equal variance. In the second, we tested McCormack et al.'s (2002) memory-distortion model. In the third, we tested a SET model. In the fourth, the distributions were assumed to be Laplacian and equal variance; however, in this model, the locations of the means of the eight equal-variance Laplace distributions were restricted to be linear with $\log _{10}[T]$, where $T$ is the duration of each stimulus. The eight parameters of this model were the locations of the seven criteria and the $S D$ of the equal-variance Laplace distributions. In the fifth, in addition to assuming a log spacing along the decision axis, we also assumed that that the criteria were fixed at the midpoints between the means of adjacent stimuli, leaving one free parameter - the $S D$ of the Laplace distributions. The $S D$ values for the young observers in this model were 0.29 and 0.30 for Experiments 1 and 2, respectively. For older observers, the corresponding values were 0.24 and 0.29 . 